Article

\title{
Two Forms of the Integral Representations of the Mittag-Leffler Function
}

\author{
Viacheslav Saenko
}

S.P. Kapitsa Research Institute of Technology, Ulyanovsk State University, L. Tolstoy St. 42, Ulyanovsk 432017,

Russia; saenkovv@gmail.com

Received: 4 June 2020; Accepted: 30 June 2020; Published: 5 July 2020

check for updates

\begin{abstract}
The integral representation of the two-parameter Mittag-Leffler function $E_{\rho, \mu}(z)$ is considered in the paper that expresses its value in terms of the contour integral. For this integral representation, the transition is made from integration over a complex variable to integration over real variables. It is shown that as a result of such a transition, the integral representation of the function $E_{\rho, \mu}(z)$ has two forms: the representation " $\mathrm{A}$ " and " $\mathrm{B}$ ". Each of these representations has its advantages and drawbacks. In the paper, the corresponding theorems are formulated and proved, and the advantages and disadvantages of each of the obtained representations are discussed.
\end{abstract}

Keywords: Mittag-Leffler function; integral representation; asymptotics

MSC: 33E12

\section{Introduction}

The Mittag-Leffler function is an entire function defined by a power series

$$
E_{\rho}(z)=\sum_{k=0}^{\infty} \frac{z^{k}}{\Gamma(1+k / \rho)}, \quad \rho>0, \quad z \in \mathbb{C},
$$

where $\Gamma(x)$ is the Gamma-function. This function was introduced by Mittag-Leffler in a number of works published from 1902 to 1905 in connection with his development of a method of summing divergent series. For more detailed information on the content of these works and on the history of the introduction of the Mittag-Leffler function, we refer the reader to the book [1] (see chapter 2 in [1]). The function itself $E_{\rho}(z)$ was introduced in the work [2]. In the paper [3], the integral representation for this function was obtained that expresses its value in terms of the contour integral.

In this paper the two parameter Mittag-Leffler function

$$
E_{\rho, \mu}(z)=\sum_{k=0}^{\infty} \frac{z^{k}}{\Gamma(\mu+k / \rho)}, \quad \rho>0, \quad \mu \in \mathbb{C}, \quad z \in \mathbb{C}
$$

is studied. This function was first introduced by A. Wiman in 1905 [4,5]. Later in 1953 this function was rediscovered in the works of Humbert and Agarval [6-8]. A new function was introduced by replacing the additive unit in the Gamma function argument in $E_{\rho}(z)$ for an arbitrary complex parameter $\mu$. At the same time, irrespective of Humbert and Agarval, the function (1) was studied by M.M. Djrbashian in the papers $[9,10]$ (see also [11] Chapter $3, \S 2,4$ ). As we can see, the two parameter Mittag-Leffler function 
$E_{\rho, \mu}(z)$ is connected with the classic Mittag-Leffler function $E_{\rho}(z)$ by a simple relation $E_{\rho, 1}(z)=E_{\rho}(z)$. For more detailed information on the properties of the function $E_{\rho, \mu}(z)$ we refer the reader to the book [1], as well as to other review works [12-14]. In this paper, integral representations of the function $E_{\rho, \mu}(z)$ will be obtained and studied.

The integral representation of the Mittag-Leffler function is important from the point of view of its practical use, as well as for studying the asymptotic properties and zeros of this function. The integral representation expressed through the contour integral is used for these purposes. Several such representations are known for the Mittag-Leffler function. One of the earliest integral representations of the function $E_{\rho, \mu}(z)$ was given in the book [15] (see §18.1, formula (20)). Further development of the issue of the integral representation of the Mittag-Leffler function and the study of its asymptotic properties was carried out in the works of M.M. Djrbashian. In the work [9] the integral representation was obtained that expressed the Mittag-Leffler function through the contour integral. Later it was included in his monograph [11] (see chapter 3, §2, Lemma 3.2.1). Using this integral representation, asymptotic formulas and the distribution of the zeros of the Mittag-Leffler function were obtained. Further, the results of the work $[9,11]$ were used in the books of [1,16], as well as in the works of [17-21] to develop the methods and algorithms of calculating the Mittag-Leffler function. However, despite a wide use of the integral representation for the Mittag-Leffler function that was obtained in the works of $[9,11]$, it turned out that there was a mistake in that representation. This fact was pointed out in the work of [22]. In this regard, there is an issue of obtaining the correct integral representation for the Mittag-Leffler function.

One of the possible solutions to this issue was given in the works of [22,23]. In these works, the representation of the Mittag-Leffler function was obtained that expresses its value through the contour integral. As it was noted earlier, this representation is used to study the asymptotic properties of the Mittag-Leffler function. However, for practical use and for calculating the value of the function, it is convenient to have integral representations expressing the function in terms of the integrals of real variables. This paper is devoted to obtaining such integral representations for the Mittag-Leffler function. The starting point of the solution to this problem is the integral representation of the function $E_{\rho, \mu}(z)$ obtained in the work [22]. Running a little ahead, we will say that the transition from integration over a complex variable to integration over real variables leads to the appearance of two forms of the integral representation of the Mittag-Leffler function. The first form will be abbreviated as the representation " $\mathrm{A}$ ", the second as the representation " $\mathrm{B}$ ". The representation " $\mathrm{A}$ " is a direct consequence of the integral representation obtained in the work [22]. It is obtained as a result of the transition from the contour integral to integration over real variables. To obtain the representation " $\mathrm{B}$ " in addition to performing such a transition, it is necessary to carry out a terminal transition $\varepsilon \rightarrow 0$. This leads to the fact that the integral representation " $\mathrm{B}$ " is valid only for parameter values $\mu$ satisfying the condition $\Re \mu<1+1 / \rho$. As a result, both the representation " $\mathrm{A}$ " and the representation " $\mathrm{B}$ " have its advantages and drawbacks which will be discussed in detail in the paper.

It should be pointed out that in this paper the letters $\rho, \mu$ are used to denote the parameters of the Mittag-Leffler function (1). These notations were introduced by M.M. Djrbashian in his works [9-11]. Parameters $\rho$ and $\mu$ of the function (1) are connected with the commonly used notations $\alpha$ and $\beta$ of the parameters of the Mittag-Leffler function by simple relations $\alpha=1 / \rho, \beta=\mu$.

\section{Integral Representation " $\mathrm{A}$ "}

The purpose of this paper is to obtain integral representations for the function $E_{\rho, \mu}(z)$ expressing this function in terms of integrals over real variables. It is convenient to use the representations of such a kind in practical problems as well as for calculating the values of the Mittag-Leffler function. As an example of the use of such integral representations, we can mention the work [24]. In that paper, the inverse Fourier 
transform of the characteristic function of the fractionally stable law was performed which is expressed in terms of the Mittag-Leffler function. To perform the inverse Fourier transform, we used the integral representation of the Mittag-Leffler function which in the current paper is formulated in Corollary 3 item 2 (see (84)). Using this integral representation in the paper [24] expressions of density and distribution function of a fractionally stable law were obtained. Articles $[25,26]$ are another example of usage of the Mittag-Leffler function. In these articles are shown that solutions of a master equation for the fractional Poisson process [25] and a fractional relaxation equation for dielectrics [26] are expressed thorough the Mittag-Leffler function. A Monte Carlo method was used for calculation the obtained solutions. However, the use of the integral representation of the Mittag-Leffler function for calculation of the solutions would significantly increase the accuracy of the results.

The starting point of this paper is the integral representation of the function $E_{\rho, u}(z)$, obtained in the work [22]. The following theorem was formulated in this work

Theorem 1. For any real $\rho, \delta_{1 \rho}, \delta_{2 \rho}, \epsilon$ satisfying the conditions $\rho>1 / 2, \frac{\pi}{2 \rho}<\delta_{1 \rho} \leqslant \min (\pi, \pi / \rho), \frac{\pi}{2 \rho}<\delta_{2 \rho} \leqslant$ $\min (\pi, \pi / \rho), \epsilon>0$, any $\mu \in \mathbb{C}$ and any $z \in \mathbb{C}$ satisfying the condition

$$
\frac{\pi}{2 \rho}-\delta_{2 \rho}+\pi<\arg z<-\frac{\pi}{2 \rho}+\delta_{1 \rho}+\pi
$$

the Mittage-Leffler function can be represented in the from

$$
E_{\rho, \mu}(z)=\frac{\rho}{2 \pi i} \int_{\gamma_{\zeta}} \frac{\exp \left\{(z \zeta)^{\rho}\right\}(z \zeta)^{\rho(1-\mu)}}{\zeta-1} d \zeta
$$

where the contour of integration $\gamma_{\zeta}$ has the form (see Figure 1)

$$
\gamma_{\zeta}=\left\{\begin{array}{l}
S_{1}=\left\{\zeta: \arg \zeta=-\delta_{1 \rho}-\pi,|\zeta| \geqslant 1+\epsilon\right\}, \\
C_{\epsilon}=\left\{\zeta:-\delta_{1 \rho}-\pi \leqslant \arg \zeta \leqslant \delta_{2 \rho}-\pi,|\zeta|=1+\epsilon\right\}, \\
S_{2}=\left\{\zeta: \arg \zeta=\delta_{2 \rho}-\pi, \quad|\zeta| \geqslant 1+\epsilon\right\} .
\end{array}\right.
$$

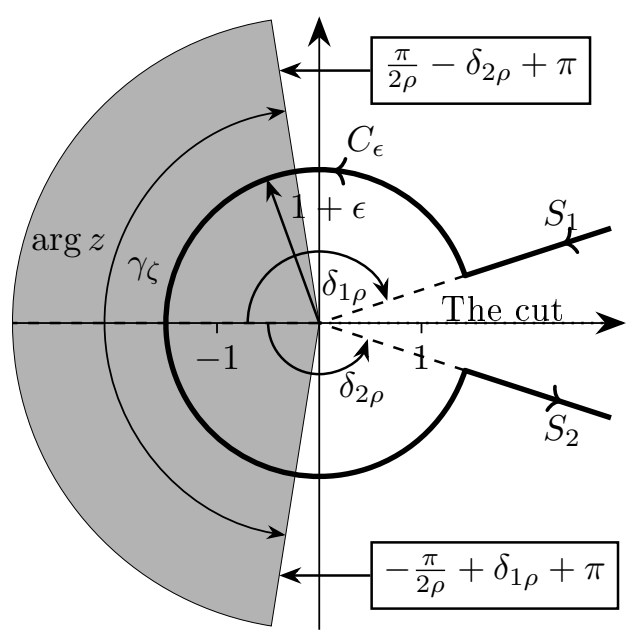

Figure 1. The contour of integration $\gamma_{\zeta}$. The region that corresponds to the condition (2) is shaded in grey.

The proof of this theorem can be found in the work [22]. 
The form of the contour of integration $\gamma_{\zeta}$ on the complex plane $\zeta$ is given in Figure 1 . The cut of the complex plane goes along the positive part of a real axis. The contour of integration consists of the half-line $S_{1}$, the arc of the circle $C_{\epsilon}$ radius $1+\epsilon$ and the half-line $S_{2}$. The contour $\gamma_{\zeta}$ is traversed in a positive direction. The parameters $\delta_{1 \rho}$ and $\delta_{2 \rho}$ have the meaning of inclination angles of the half-lines $S_{1}$ and $S_{2}$ in relation to the contour axis $\gamma_{\zeta}$. In Figure 1 the contour axis $\gamma_{\zeta}$ coincides with a real axis. The values of the angles $\delta_{1 \rho}$ and $\delta_{2 \rho}$ are measured from the negative part of the real axis. The reference directions of these angles in Figure 1 are shown by arrows.

We will obtain the integral representations that of interest to us by going from integration over the complex variable $\zeta$ to integration over variables $r$ and $\varphi$ interconnected by the relation $\zeta=r e^{i \varphi}$. As a result, the following theorem is true for the function $E_{\rho, \mu}(z)$.

Theorem 2 (The representation " $\mathrm{A}$ "). For any real $\rho>1 / 2, \epsilon>0$ and $\delta_{1 \rho}, \delta_{2 \rho}$ satisfying the conditions

$$
\frac{\pi}{2 \rho}<\delta_{1 \rho} \leqslant \min \left(\pi, \frac{\pi}{\rho}\right), \quad \frac{\pi}{2 \rho}<\delta_{2 \rho} \leqslant \min \left(\pi, \frac{\pi}{\rho}\right)
$$

for any complex $\mu$ and any complex $z$ satisfying the condition

$$
\frac{\pi}{2 \rho}-\delta_{2 \rho}+\pi<\arg z<-\frac{\pi}{2 \rho}+\delta_{1 \rho}+\pi,
$$

the Mittag-Leffler function can be represented in the form

$$
E_{\rho, \mu}(z)=\int_{1+\epsilon}^{\infty} K_{\rho, \mu}\left(r,-\delta_{1 \rho}, \delta_{2 \rho}, z\right) d r+\int_{-\delta_{1 \rho}-\pi}^{\delta_{2 \rho}-\pi} P_{\rho, \mu}(1+\epsilon, \varphi, z) d \varphi .
$$

Here

$$
K_{\rho, \mu}\left(r, \varphi_{1}, \varphi_{2}, z\right)=\frac{\rho}{2 \pi i} \frac{(z r)^{\rho(1-\mu)} e^{-i \rho \pi(1-\mu)}\left[A_{\rho, \mu}\left(r, \varphi_{2}, \varphi_{1}, z\right)-A_{\rho, \mu}\left(r, \varphi_{1}, \varphi_{2}, z\right)\right]}{\left(r^{2}+2 r \cos \varphi_{1}+1\right)\left(r^{2}+2 r \cos \varphi_{2}+1\right)}
$$

where

$$
\begin{gathered}
A_{\rho, \mu}\left(r, \omega_{1}, \omega_{2}, z\right)=\exp \left\{(z r)^{\rho} e^{-i \rho \pi} \cos \left(\rho \omega_{1}\right)\right\}\left(r^{2}+2 r \cos \omega_{2}+1\right) e^{i \eta\left(r, \omega_{1}, z\right)}\left[r+e^{i \omega_{1}}\right], \\
\eta(r, \varphi, z)=(z r)^{\rho} e^{-i \rho \pi} \sin (\rho \varphi)+\rho(1-\mu) \varphi,
\end{gathered}
$$

and

$$
P_{\rho, \mu}(r, \varphi, z)=\frac{\rho}{2 \pi} \frac{\exp \left\{(z r)^{\rho} \cos (\rho \varphi)\right\}(z r)^{\rho(1-\mu)} e^{i \chi(r, \varphi, z)} r\left[r-e^{i \varphi}\right]}{r^{2}-2 r \cos \varphi+1},
$$

where

$$
\chi(r, \varphi, z)=(z r)^{\rho} \sin (\rho \varphi)+\rho(1-\mu) \varphi .
$$

Proof. For convenience we will introduce the notation

$$
\phi_{\rho, \mu}(\zeta, z)=\frac{\rho}{2 \pi i} \exp \left\{(\zeta z)^{\rho}\right\}(\zeta z)^{\rho(1-\mu)} .
$$


As a result, the representation (3) will take the form

$$
E_{\rho, \mu}(z)=\int_{\gamma_{\zeta}} \frac{\phi_{\rho, \mu}(\zeta, z)}{\zeta-1} d \zeta
$$

where the contour of integration $\gamma_{\zeta}$ is defined by (4).

Substituting in (14) the variable of integration $\zeta=r e^{i \varphi}$ and directly calculating this integral we obtain

$$
\begin{array}{r}
E_{\rho, \mu}(z)=\int_{S_{1}} \frac{\phi_{\rho, \mu}(\zeta, z)}{\zeta-1} d \zeta+\int_{C_{\epsilon}} \frac{\phi_{\rho, \mu}(\zeta, z)}{\zeta-1} d \zeta+\int_{S_{2}} \frac{\phi_{\rho, \mu}(\zeta, z)}{\zeta-1} d \zeta=\left.\int_{\infty}^{1+\epsilon} \frac{\phi_{\rho, \mu}\left(r e^{i \varphi}, z\right)}{r e^{i \varphi}-1} e^{i \varphi} d r\right|_{\varphi=-\delta_{1 \rho}-\pi} \\
+\left.i \int_{-\delta_{1 \rho}-\pi}^{\delta_{2 \rho}-\pi} \frac{\phi_{\rho, \mu}\left(r e^{i \varphi}, z\right)}{r e^{i \varphi}-1} r e^{i \varphi} d \varphi\right|_{r=1+\epsilon} \quad+\left.\int_{1+\epsilon}^{\infty} \frac{\phi_{\rho, \mu}\left(r e^{i \varphi}, z\right)}{r e^{i \varphi}-1} e^{i \varphi} d r\right|_{\varphi=\delta_{2 \rho}-\pi}=\int_{\infty}^{1+\epsilon} \frac{\phi_{\rho, \mu}\left(r e^{i\left(-\delta_{1 \rho}-\pi\right)}, z\right)}{r e^{i\left(-\delta_{1 \rho}-\pi\right)}-1} e^{i\left(-\delta_{1 \rho}-\pi\right)} d r \\
+i \int_{-\delta_{1 \rho}-\pi}^{\delta_{2 \rho}-\pi} \frac{\phi_{\rho, \mu}\left((1+\epsilon) e^{i \varphi}, z\right)}{(1+\epsilon) e^{i \varphi}-1}(1+\epsilon) e^{i \varphi} d \varphi+\int_{1+\epsilon}^{\infty} \frac{\phi_{\rho, \mu}\left(r e^{i\left(\delta_{2 \rho}-\pi\right)}, z\right)}{r e^{i\left(\delta_{2 \rho}-\pi\right)}-1} e^{i\left(\delta_{2 \rho}-\pi\right)} d r=I_{S_{1}}+I_{C_{\epsilon}}+I_{S_{2}} .
\end{array}
$$

We consider the integral $I_{S_{1}}$. Getting rid of the complexity in the denominator we have

$$
\begin{gathered}
I_{S_{1}}=\int_{\infty}^{1+\epsilon} \frac{\phi_{\rho, \mu}\left(r e^{i\left(-\delta_{1 \rho}-\pi\right)}, z\right) e^{i\left(-\delta_{1 \rho}-\pi\right)}}{r e^{i\left(-\delta_{1 \rho}-\pi\right)}-1} d r=\int_{\infty}^{1+\epsilon} \frac{\phi_{\rho, \mu}\left(r e^{i\left(-\delta_{1 \rho}-\pi\right)}, z\right) e^{i\left(-\delta_{1 \rho}-\pi\right)}\left(r e^{i\left(\delta_{1 \rho}+\pi\right)}-1\right)}{\left(r e^{i\left(-\delta_{1 \rho}-\pi\right)}-1\right)\left(r e^{i\left(\delta_{1 \rho}+\pi\right)}-1\right)} d r \\
=\int_{\infty}^{1+\epsilon} \frac{\phi_{\rho, \mu}\left(r e^{i\left(-\delta_{1 \rho}-\pi\right)}, z\right)\left(r+e^{-i \delta_{1 \rho}}\right)}{r^{2}+2 r \cos \delta_{1 \rho}+1} d r \\
=\frac{\rho}{2 \pi i} \int_{\infty}^{1+\epsilon} \frac{\exp \left\{\left(r z e^{-i\left(\delta_{1 \rho}+\pi\right)}\right)^{\rho}\right\}\left(z r e^{-i\left(\delta_{1 \rho}+\pi\right)}\right)^{\rho(1-\mu)}\left(r+e^{-i \delta_{1 \rho}}\right)}{r^{2}+2 r \cos \delta_{1 \rho}+1} d r \\
=\frac{\rho}{2 \pi i} \int_{\infty}^{1+\epsilon} \frac{\exp \left\{(z r)^{\rho} e^{-i \rho \pi}\left(\cos \left(\rho \delta_{1 \rho}\right)-i \sin \left(\rho \delta_{1 \rho}\right)\right)\right\}}{r^{2}+2 r \cos \delta_{1 \rho}+1}(z r)^{\rho(1-\mu)} e^{-i \rho \pi(1-\mu)} e^{-i \rho(1-\mu) \delta_{1 \rho}}\left(r+e^{-i \delta_{1 \rho}}\right) d r \\
=\frac{\rho}{2 \pi i} \int_{\infty}^{1+\epsilon} \frac{\exp \left\{(z r)^{\rho} e^{-i \rho \pi} \cos \left(\rho \delta_{1 \rho}\right)\right\}}{r^{2}+2 r \cos \delta_{1 \rho}+1}(z r)^{\rho(1-\mu)} e^{-i \rho \pi(1-\mu)} e^{i \eta\left(r,-\delta_{1 \rho}, z\right)}\left(r+e^{-i \delta_{1 \rho}}\right) d r
\end{gathered}
$$

where the notation $\eta(r, \varphi, z)=(z r)^{\rho} e^{-i \rho \pi} \sin (\rho \varphi)+\rho(1-\mu) \varphi$ was introduced.

Similarly, for the integral $I_{S_{2}}$ we get

$$
\begin{gathered}
I_{S_{2}}=\int_{1+\epsilon}^{\infty} \frac{\phi_{\rho, \mu}\left(r e^{i\left(\delta_{2 \rho}-\pi\right)}, z\right) e^{i\left(\delta_{2 \rho}-\pi\right)}}{r e^{i\left(\delta_{2 \rho}-\pi\right)}-1} d r=\int_{1+\epsilon}^{\infty} \frac{\phi_{\rho, \mu}\left(r e^{i\left(\delta_{2 \rho}-\pi\right)}, z\right)\left(r+e^{i \delta_{2 \rho}}\right)}{r^{2}+2 r \cos \delta_{2 \rho}+1} d r \\
=\frac{\rho}{2 \pi i} \int_{1+\epsilon}^{\infty} \frac{\exp \left\{(z r)^{\rho} e^{-i \rho \pi} \cos \left(\rho \delta_{2 \rho}\right)\right\}(z r)^{\rho(1-\mu)} e^{-i \rho \pi(1-\mu)} e^{i \eta\left(r, \delta_{2 \rho}, z\right)}\left(r+e^{i \delta_{2 \rho}}\right)}{r^{2}+2 r \cos \delta_{2 \rho}+1} d r
\end{gathered}
$$


Summing now the integrals $I_{S_{1}}$ and $I_{S_{2}}$ we obtain

$$
\begin{aligned}
I_{S_{1}}+I_{S_{2}}= & \frac{\rho}{2 \pi i} \int_{\infty}^{1+\epsilon} \frac{\exp \left\{(z r)^{\rho} e^{-i \rho \pi} \cos \left(\rho \delta_{1 \rho}\right)\right\}}{r^{2}+2 r \cos \delta_{1 \rho}+1}(z r)^{\rho(1-\mu)} e^{-i \rho \pi(1-\mu)} e^{i \eta\left(r,-\delta_{1 \rho}, z\right)}\left(r+e^{-i \delta_{1 \rho}}\right) d r \\
& +\frac{\rho}{2 \pi i} \int_{1+\epsilon}^{\infty} \frac{\exp \left\{(z r)^{\rho} e^{-i \rho \pi} \cos \left(\rho \delta_{2 \rho}\right)\right\}(z r)^{\rho(1-\mu)} e^{-i \rho \pi(1-\mu)} e^{i \eta\left(r, \delta_{2 \rho}, z\right)}\left(r+e^{i \delta_{2 \rho}}\right)}{r^{2}+2 r \cos \delta_{2 \rho}+1} d r \\
& =\int_{1+\epsilon}^{\infty} K_{\rho, \mu}\left(r,-\delta_{1 \rho}, \delta_{2 \rho}, z\right) d r,
\end{aligned}
$$

where

$$
\begin{aligned}
K_{\rho, \mu}\left(r, \varphi_{1}, \varphi_{2}, z\right) & =\frac{\rho}{2 \pi i} \frac{(z r)^{\rho(1-\mu)} e^{-i \rho \pi(1-\mu)}\left[A_{\rho, \mu}\left(r, \varphi_{2}, \varphi_{1}, z\right)-A_{\rho, \mu}\left(r, \varphi_{1}, \varphi_{2}, z\right)\right]}{\left(r^{2}+2 r \cos \varphi_{1}+1\right)\left(r^{2}+2 r \cos \varphi_{2}+1\right)}, \\
A_{\rho, \mu}\left(r, \omega_{1}, \omega_{2}, z\right) & =\exp \left\{(z r)^{\rho} e^{-i \rho \pi} \cos \left(\rho \omega_{1}\right)\right\}\left(r^{2}+2 r \cos \omega_{2}+1\right) e^{i \eta\left(r, \omega_{1}, z\right)}\left[r+e^{i \omega_{1}}\right] .
\end{aligned}
$$

We consider now the integral $I_{C_{e}}$. At the beginning, we will get rid of the complexity in the denominator. To do this, we will multiply and divide the integrand by the complex conjugate of the denominator and open the brackets in the denominator. Then, in the resulting expression, we substitute the definition of the function $\phi_{\rho, \mu}(\zeta, z)$ (see (13)) and in the indices of the exponents we use the Euler formula $e^{i \varphi}=\cos \varphi+i \sin \varphi$. As a result, we get

$$
\begin{gathered}
I_{C_{\epsilon}}=i \int_{-\delta_{1 \rho}-\pi}^{\delta_{2 \rho}-\pi} \frac{\phi_{\rho, \mu}\left((1+\epsilon) e^{i \varphi}, z\right)}{(1+\epsilon) e^{i \varphi}-1}(1+\epsilon) e^{i \varphi} d \varphi=i \int_{-\delta_{1 \rho}-\pi}^{\delta_{2 \rho}-\pi} \frac{\phi_{\rho, \mu}\left((1+\epsilon) e^{i \varphi}, z\right)(1+\epsilon) e^{i \varphi}\left((1+\epsilon) e^{-i \varphi}-1\right)}{\left((1+\epsilon) e^{i \varphi}-1\right)\left((1+\epsilon) e^{-i \varphi}-1\right)} d \varphi \\
=i \int_{-\delta_{1 \rho}-\pi}^{\delta_{2 \rho}-\pi} \frac{\phi_{\rho, \mu}\left((1+\epsilon) e^{i \varphi}, z\right)(1+\epsilon)\left((1+\epsilon)-e^{i \varphi}\right)}{(1+\epsilon)^{2}-2(1+\epsilon) \cos \varphi+1} d \varphi \\
=\frac{\rho}{2 \pi} \int_{-\delta_{1 \rho}-\pi}^{\delta_{2 \rho}-\pi} \frac{\exp \left\{\left(z(1+\epsilon) e^{i \varphi}\right)^{\rho}\right\}\left(z(1+\epsilon) e^{i \varphi}\right)^{\rho(1-\mu)}(1+\epsilon)\left((1+\epsilon)-e^{i \varphi}\right)}{(1+\epsilon)^{2}-2(1+\epsilon) \cos \varphi+1} d \varphi \\
=\frac{\rho}{2 \pi} \int_{-\delta_{1 \rho}-\pi}^{\delta_{2 \rho}-\pi} \frac{\exp \left\{((1+\epsilon) z)^{\rho}(\cos (\rho \varphi)+i \sin (\rho \varphi))\right\}}{(1+\epsilon)^{2}-2(1+\epsilon) \cos \varphi+1}(z(1+\epsilon))^{\rho(1-\mu)} e^{i \rho \varphi(1-\mu)}(1+\epsilon)\left((1+\epsilon)-e^{i \varphi}\right) d \varphi \\
=\frac{\rho}{2 \pi} \int_{-\delta_{1 \rho}-\pi}^{\delta_{2 \rho}-\pi} \frac{\exp \left\{((1+\epsilon) z)^{\rho} \cos (\rho \varphi)\right\}}{(1+\epsilon)^{2}-2(1+\epsilon) \cos \varphi+1}(z(1+\epsilon))^{\rho(1-\mu)} e^{i \chi((1+\epsilon), \varphi, z)}(1+\epsilon)\left((1+\epsilon)-e^{i \varphi}\right) d \varphi \\
=\int_{-\delta_{1 \rho}-\pi}^{\delta_{2 \rho}-\pi} P_{\rho, \mu}(1+\epsilon, \varphi, z) d \varphi,
\end{gathered}
$$

where

$$
\begin{aligned}
P_{\rho, \mu}(r, \varphi, z) & =\frac{\rho}{2 \pi} \frac{\exp \left\{(z r)^{\rho} \cos (\rho \varphi)\right\}(z r)^{\rho(1-\mu)} e^{i \chi(r, \varphi, z)} r\left[r-e^{i \varphi}\right]}{r^{2}-2 r \cos \varphi+1} \\
\chi(r, \varphi, z) & =(z r)^{\rho} \sin (\rho \varphi)+\rho(1-\mu) \varphi .
\end{aligned}
$$

Using (16) and (17) in (15) we obtain the representation (7). It is important to pay attention that in the process of proving no additional limitations on the values of parameters $\rho, \mu, \delta_{1 \rho}, \delta_{2 \rho}$ and the argument $z$ 
were imposed and it means that the ranges of admissible values pass from Theorem 1 without change. Thus, the representation (7) is valid for any real $\rho>1 / 2$, any real $\delta_{1 \rho}, \delta_{2 \rho}$ satisfying the conditions (5), any complex $\mu$ and any complex $z$ satisfying the condition (6).

The proved theorem formulates an integral representation for the Mittag-Leffler function that expresses this function in terms of the sum of improper and definite integrals. To be definite, we will call this integral representation of the Mittag-Leffler function the representation " $A$ ". As we can see, the representation " $\mathrm{A}$ " is a direct consequence of the representation (3). It is obtained by passing from the contour integral to integrals over the real variable. Moreover, the improper integral in (7) corresponds to the sum of integrals along the half-lines $S_{1}$ and $S_{2}$ of the contour $\gamma_{\zeta}$ and the definite integral corresponds to the integral along the arc of a circle $C_{\epsilon}$. It should be noted that this integral is taken along the arc of a circle of radius $1+\epsilon$, where $\epsilon>0$. The representation (7) is valid for arbitrary values $\rho>1 / 2$, any $\mu$ and any $\delta_{1 \rho}$ and $\delta_{2 \rho}$ that satisfy the condition (5).

However, in general case, for arbitrary values of parameters $\delta_{1 \rho}$ and $\delta_{2 \rho}$, satisfying the condition (5), the kernel function $K_{\rho, \mu}\left(r,-\delta_{1 \rho}, \delta_{2 \rho}, z\right)$ turns to be lengthy. The representation (7) takes the more compact form in case when the half-lines $S_{1}$ and $S_{2}$ of the contour of integration $\gamma_{\zeta}$ run symmetrically relative to the real axis i.e., when $\delta_{1 \rho}=\delta_{2 \rho}=\delta_{\rho}$. We will formulate the obtained result in the form of a corollary.

Corollary 1. For any real $\epsilon>0$, any complex $\mu$ the following integral representations of the Mittag-Leffler function are true:

1. at any real $\rho>1 / 2$, any real $\delta_{\rho}$ satisfying the condition $\frac{\pi}{2 \rho}<\delta_{\rho} \leqslant \min \left(\pi, \frac{\pi}{\rho}\right)$ and any complex $z$ satisfying the condition $\frac{\pi}{2 \rho}-\delta_{\rho}+\pi<\arg z<-\frac{\pi}{2 \rho}+\delta_{\rho}+\pi$

$$
E_{\rho, \mu}(z)=\int_{1+\epsilon}^{\infty} K_{\rho, \mu}\left(r, \delta_{\rho}, z\right) d r+\int_{-\delta_{\rho}-\pi}^{\delta_{\rho}-\pi} P_{\rho, \mu}(1+\epsilon, \varphi, z) d \varphi,
$$

where

$$
K_{\rho, \mu}(r, \varphi, z)=\frac{\rho}{\pi}\left(z r e^{-i \pi}\right)^{\rho(1-\mu)} \exp \left\{\left(z r e^{-i \pi}\right)^{\rho} \cos (\rho \varphi)\right\} \frac{r \sin (\eta(r, \varphi, z))+\sin (\eta(r, \varphi, z)+\varphi)}{r^{2}+2 r \cos \varphi+1},
$$

$\eta(r, \varphi, z)$ is defined by (10) and $P_{\rho, \mu}(r, \varphi, z)$ has the form (11).

2. at any real $\rho \geqslant 1$ at $\delta_{\rho}=\pi / \rho$ and any complex $z$ satisfying the condition $-\frac{\pi}{2 \rho}+\pi<\arg z<\frac{\pi}{2 \rho}+\pi$

$$
E_{\rho, \mu}(z)=\int_{1+\epsilon}^{\infty} K_{\rho, \mu}(r, z) d r+\int_{-\frac{\pi}{\rho}-\pi}^{\frac{\pi}{\rho}-\pi} P_{\rho, \mu}(1+\epsilon, \varphi, z) d \varphi,
$$

where

$$
K_{\rho, \mu}(r, z)=\frac{\rho}{\pi}\left(z r e^{-i \pi}\right)^{\rho(1-\mu)} \exp \left\{-\left(z r e^{-i \pi}\right)^{\rho}\right\} \frac{r \sin (\pi(1-\mu))+\sin (\pi(1-\mu)+\pi / \rho)}{r^{2}+2 r \cos (\pi / \rho)+1}
$$

and $P_{\rho, \mu}(r, \varphi, z)$ is defined by (11).

Proof. (1) According to Theorem 2, the Mittag-Leffler function can be represented in the form (7). This representation is true for arbitrary $\delta_{1 \rho}$ and $\delta_{2 \rho}$ satisfying the conditions (5). In case if $\delta_{1 \rho}=\delta_{2 \rho}=\delta_{\rho}$ the conditions (5) take the form

$$
\frac{\pi}{2 \rho}<\delta_{\rho} \leqslant \min \left(\pi, \frac{\pi}{\rho}\right)
$$


and the condition (6) can be written in the form

$$
\frac{\pi}{2 \rho}-\delta_{\rho}+\pi<\arg z<-\frac{\pi}{2 \rho}+\delta_{\rho}+\pi
$$

As a result, the Mittag-Leffler function is written in the form

$$
E_{\rho, \mu}(z)=\int_{1+\epsilon}^{\infty} K_{\rho, \mu}\left(r,-\delta_{\rho}, \delta_{\rho}, z\right) d r+\int_{-\delta_{\rho}-\pi}^{\delta_{\rho}-\pi} P_{\rho, \mu}(1+\epsilon, \varphi, z) d \varphi .
$$

We consider the integrand of the first integral and denote

$$
K_{\rho, \mu}\left(r, \delta_{\rho}, z\right) \equiv K_{\rho, \mu}\left(r,-\delta_{\rho}, \delta_{\rho}, z\right)
$$

Using the definition $K_{\rho, \mu}\left(r, \varphi_{1}, \varphi_{2}, z\right)$ (see (8)) we get

$$
\begin{aligned}
& K_{\rho, \mu}\left(r, \delta_{\rho}, z\right)=K_{\rho, \mu}\left(r,-\delta_{\rho}, \delta_{\rho}, z\right) \\
&=\frac{\rho}{2 \pi i} \frac{(z r)^{\rho(1-\mu)} e^{-i \rho \pi(1-\mu)}\left(A_{\rho, \mu}\left(r, \delta_{\rho},-\delta_{\rho}, z\right)-A_{\rho, \mu}\left(r,-\delta_{\rho}, \delta_{\rho}, z\right)\right)}{\left(r^{2}+2 r \cos \delta_{\rho}+1\right)^{2}} .
\end{aligned}
$$

Using the definition $A_{\rho, \mu}\left(r, \omega_{1}, \omega_{2}, z\right)$ (see (9)) and the fact that the function $\eta(r, \varphi, z)$ defined by (10) is an odd function according to the variable $\varphi$

$$
\eta(r,-\varphi, z)=-\eta(r, \varphi, z)
$$

we have

$$
\begin{aligned}
A_{\rho, \mu}\left(r, \delta_{\rho},-\delta_{\rho}, z\right)- & A_{\rho, \mu}\left(r,-\delta_{\rho}, \delta_{\rho}, z\right) \\
& =\exp \left\{(z r)^{\rho} e^{-i \pi \rho} \cos \left(\rho \delta_{\rho}\right)\right\}\left(r^{2}+2 r \cos \left(-\delta_{\rho}\right)+1\right) e^{i \eta\left(r, \delta_{\rho}, z\right)}\left(r+e^{i \delta_{\rho}}\right) \\
& -\exp \left\{(z r)^{\rho} e^{-i \pi \rho} \cos \left(-\rho \delta_{\rho}\right)\right\}\left(r^{2}+2 r \cos \delta_{\rho}+1\right) e^{i \eta\left(r,-\delta_{\rho}, z\right)}\left(r+e^{-i \delta_{\rho}}\right) \\
=\exp \left\{(z r)^{\rho} e^{-i \pi \rho}\right. & \left.\cos \left(\rho \delta_{\rho}\right)\right\}\left(r^{2}+2 r \cos \delta_{\rho}+1\right)\left(r e^{i \eta\left(r, \delta_{\rho}, z\right)}+e^{i\left(\eta\left(r, \delta_{\rho}, z\right)+\delta_{\rho}\right)}-r e^{-i \eta\left(r, \delta_{\rho}, z\right)}-e^{-i\left(\eta\left(r, \delta_{\rho}, z\right)+\delta_{\rho}\right)}\right) \\
= & 2 i \exp \left\{(z r)^{\rho} e^{-i \pi \rho} \cos \left(\rho \delta_{\rho}\right)\right\}\left(r^{2}+2 r \cos \delta_{\rho}+1\right)\left(r \sin \left(\eta\left(r, \delta_{\rho}, z\right)\right)+\sin \left(\eta\left(r, \delta_{\rho}, z\right)+\delta_{\rho}\right)\right) .
\end{aligned}
$$

Now substituting this result in (25) we obtain (19). Since in the proof process no additional restrictions on the values of parameters $\rho, \mu$ and on the value $\arg z$ were imposed, then the conditions for these parameters go from Theorem 2 without change. Thus, we come to the conditions of the corollary.

(2) We consider the case $\delta_{1 \rho}=\delta_{2 \rho}=\pi / \rho$. As we can see, the case considered is a particular case of the previous one. It follows from (5) that this case can be implemented if $\rho \geqslant 1$. For the range of values $\arg z$ from (23) we get

$$
-\frac{\pi}{2 \rho}+\pi<\arg z<\frac{\pi}{2 \rho}+\pi
$$

Now we consider the representation (18). In the case under consideration it will be written in the form

$$
E_{\rho, \mu}(z)=\int_{1+\epsilon}^{\infty} K_{\rho, \mu}(r, \pi / \rho, z) d r+\int_{-\frac{\pi}{\rho}-\pi}^{\frac{\pi}{\rho}-\pi} P_{\rho, \mu}(1+\epsilon, \varphi, z) d \varphi .
$$


We denote

$$
K_{\rho, \mu}(r, z) \equiv K_{\rho, \mu}\left(r, \frac{\pi}{\rho}, z\right) .
$$

From (10) it follows that $\eta\left(r, \frac{\pi}{\rho}, z\right)=(1-\mu) \pi$. Now using this result in (19) we get (21).

From the proved corollary it follows that if the parameter values $\delta_{1 \rho}$ and $\delta_{2 \rho}$ coincide, then in this the kernel function $K_{\rho, \mu}\left(r, \varphi_{1}, \varphi_{2}, z\right)$ is significantly simplified. Recall that the parameters $\delta_{1 \rho}$ and $\delta_{2 \rho}$ are inclination angles of half-lines $S_{1}$ and $S_{2}$ in the contour $\gamma_{\zeta}$ relative to the axis of this contour (see Figure 1). Since in the Theorem 1 the axis of the contour $\gamma_{\zeta}$ coincides with the real axis, then the selection of $\delta_{1 \rho}=\delta_{2 \rho}$ means that half-lines $S_{1}$ and $S_{2}$ run symmetrically in relation to the real axis. The kernel function $K_{\rho, \mu}\left(r, \varphi_{1}, \varphi_{2}, z\right)$ takes the simplest form in the case $\delta_{1 \rho}=\delta_{2 \rho}=\pi / \rho$.

Further, it is necessary for us to know the position of singular points of the integrand of the representation (3). This issue was studied in the work [27]. For completeness of the statement here we give the result obtained in the work [27] and formulate it in the form of a lemma.

Lemma 1. For any real $\rho>1 / 2$ and any complex values of the parameter $\mu=\mu_{R}+i \mu_{I}$ the integrand of the representation (3)

$$
\Phi_{\rho, \mu}(\zeta, z)=\frac{\rho}{2 \pi i} \frac{\exp \left\{(\zeta z)^{\rho}\right\}(\zeta z)^{\rho(1-\mu)}}{\zeta-1} .
$$

relative to the variable $\zeta$ has two singular points $\zeta=1$ and $\zeta=0$. The point $\zeta=1$ is a pole of the first order. The point $\zeta=0$ is:

1. the regular point of the function $\Phi_{\rho, \mu}(\zeta, z)$, with the values of parameters $\rho=n$, where $n=1,2,3, \ldots$ (the positive integer), $\mu_{I}=0$ and $\mu_{R}=1-m_{1} / \rho$, where $m_{1}=0,1,2,3, \ldots$ (the non-negative integer);

2. a pole of the order $m_{2}$, if $\rho=n$, where $n=1,2,3, \ldots$ (the positive integer), $\mu_{I}=0$ and $\mu_{R}=1+m_{2} / \rho$, where $m_{2}=1,2,3, \ldots$ (the positive integer);

3. the branch point, for any other values of parameters $\rho, \mu_{I}, \mu_{R}$.

The proof of this lemma can be found in the work [27].

We will make the following remark to Corollary 1.

Remark 1. In Corollary 1 the special case under consideration $\delta_{1 \rho}=\delta_{2 \rho}=\pi / \rho$. We will assume that $\rho=1$ and study the behavior of the Formula (20) in this case. As a result, we obtain

$$
E_{1, \mu}(z)=\int_{1+\epsilon}^{\infty} K_{1, \mu}(r, z) d r+\int_{-2 \pi}^{0} P_{1, \mu}(1+\epsilon, \varphi, z) d \varphi .
$$

Using (21) for $K_{1, \mu}(z)$ we obtain

$$
\begin{aligned}
K_{1, \mu}(r, z)=\frac{1}{\pi}\left(z r e^{-i \pi}\right)^{1-\mu} \exp \left\{-z r e^{-i \pi}\right\} \frac{r \sin (\pi(1-\mu))+\sin (\pi(1-\mu)+\pi)}{r^{2}-2 r+1}= \\
\frac{1}{\pi}(-z)^{1-\mu} e^{z r} \frac{\sin (\pi(1-\mu))(r-1)}{(r-1)^{2}}=\frac{1}{\pi}(-z)^{1-\mu} e^{z r} \frac{\sin (\pi(1-\mu))}{r-1} .
\end{aligned}
$$

From here it is clear, if $\mu=n$, where $n=0, \pm 1, \pm 2, \pm 3, \ldots$, then $\sin (\pi(1-\mu))=0$. Consequently,

$$
K_{1, \rho}(z)=\left\{\begin{array}{cl}
0, & \mu=n, \\
\frac{1}{\pi}(-z)^{1-\mu} e^{z r \frac{\sin (\pi(1-\mu))}{r-1},} & \mu \neq n .
\end{array}\right.
$$


Thus, with integer values of $\mu$ the first summand in (28) becomes zero and to calculate the value of $E_{1, n}(z)$ it remains to calculate the second integral. To calculate this integral, numerical methods can be used. However, in this case, this integral can be calculated analytically using the residue theory.

In fact, we return to the integral representation formulated in Theorem 1. Recall that we consider the case $\rho=1$. Using the notation (27), the representation (3) takes the form

$$
E_{1, \mu}(z)=\int_{\gamma_{\zeta}} \Phi_{1, \mu}(\zeta, z) d \zeta
$$

where the contour of integration $\gamma_{\zeta}$, defined by $(4)$, is written in the form

$$
\gamma_{\zeta}=\left\{\begin{array}{l}
S_{1}=\{\zeta: \arg \zeta=-2 \pi,|\zeta| \geqslant 1+\epsilon\}, \\
C_{\epsilon}=\{\zeta:-2 \pi \leqslant \arg \zeta \leqslant 0,|\zeta|=1+\epsilon\}, \\
S_{2}=\{\zeta: \arg \zeta=0, \quad|\zeta| \geqslant 1+\epsilon\} .
\end{array}\right.
$$

We represent the complex parameter $\mu$ in the form $\mu=\mu_{R}+i \mu_{I}$ and make use of Lemma 1. According to this lemma, the function $\Phi_{1, \mu}(\zeta, z)$ at values $\mu_{I}=0$ and $\mu_{R}=1-m_{1}$, where $m_{1}=0,1,2,3, \ldots$ has one singular point $\zeta=1$ which is a pole of the first order. The point $\zeta=0$, in this case, is the regular point. In case, if $\mu_{I}=0$ and $\mu_{R}=1+m_{2}$, where $m_{2}=1,2,3, \ldots$ the function $\Phi_{1, \mu}(\zeta, z)$ has two singular points: the point $\zeta=1$ is a pole of the first order and the point $\zeta=0$ is a pole of the order $m_{2}$. As we can see, in both cases the point $\zeta=0$ is not a branch point. As a result, in these two cases, the function $\Phi_{1, u}(\zeta, z)$ is the entire function of a complex variable $\zeta$. From here it follows that when $\mu_{I}=0$, and $\mu_{R}=n$, where $n=0, \pm 1, \pm 2, \pm 3, \ldots$ the arc of the circle $C_{\epsilon}$ that enters the contour (31) is the closed circle of radius $1+\epsilon$. The half-lines $S_{1}$ and $S_{2}$ pass along the positive part of a real axis in mutually opposite directions. With all other values of the parameter $\mu$ (when $\mu_{I} \neq 0$ or $\mu_{R} \neq n$ ), according to Lemma 1, the point $\zeta=0$ is a branch point of the function $\Phi_{1, \mu}(\zeta, z)$. In this case, the circle $C_{\epsilon}$ of the contour (31) will not close up and half-lines $S_{1}$ and $S_{2}$ will go along the upper and lower banks of the cut of the complex plane which runs along the positive part of a real axis.

It is clear from here that the result (29) is a consequence of Lemma 1 In fact, in the case when the parameter $\mu$ takes integer real values, the first and second items of Lemma 1 turn out to be true. As we have already pointed out, in this case the arc of the circle $C_{\epsilon}$ of the contour (31) is a closed circle and the half-lines $S_{1}$ and $S_{2}$ run along the positive part of a real axis in mutually opposite directions. Consequently, the sum of the integrals along the half-lines $S_{1}$ and $S_{2}$ will be equal to zero. Next, it is necessary to recall that the improper integral in the expression (28) just corresponds to the sum of the integrals along the half-lines $S_{1}$ and $S_{2}$. Therefore, with integer real values $\mu$ it should be equal to zero which has been obtained. A definite integral in (28) corresponds to integration along the closed circle. Therefore, one can use the theory of residues to calculate it.

The calculation of the integral in (30) using the theory of residues with integer real values of the parameter $\mu$ was carried out in the work [27]. For completeness of the statement, we will give the results obtained in this paper and formulate them in the form of a corollary to Lemma 1.

Corollary 2. For the values of the parameters $\rho=1, \delta_{1 \rho}=\delta_{2 \rho}=\pi$, any complex $z$, satisfying the condition $\pi / 2<\arg z<3 \pi / 2$ and for integer real values of the parameter $\mu=n, n=0, \pm 1, \pm 2, \pm 3, \ldots$ the Mittag-Leffler function has the form:

1. if $n \leqslant 1$ (i.e., $n=1,0,-1,-2,-3, \ldots$ ), then

$$
E_{1, n}(z)=e^{z} z^{1-n}
$$


2. if $n \geqslant 2($ i.e., $n=2,3,4, \ldots)$, then

$$
E_{1, n}(z)=z^{1-n}\left(e^{z}-\sum_{k=0}^{n-2} \frac{z^{k}}{k !}\right)
$$

The proof of this corollary can be found in the paper [27].

\section{Integral Representation " $B$ "}

The integral representation " $\mathrm{A}$ " consists of the sum of two integrals. As it has been found earlier, the improper integral in (7) corresponds to the sum of integrals along the half-lines $S_{1}$ and $S_{2}$ of the contour $\gamma_{\zeta}$ in the representation (3), a definite integral corresponds to the integral along the arc of the circle $C_{\epsilon}$. As a result, in analytical studies of the function $E_{\rho, \mu}(z)$, as well as in the solution of problems where it is encountered, one should conduct studies of these two integrals. This causes certain difficulties. As it will be shown below in the representation (7) one can get rid of an integral on the arc of the circle $C_{\epsilon}$ and write the integral representation for the function $E_{\rho, \mu}(z)$ in the form of an improper integral. This representation will be much easier to use. However, as a result of such a transition, some restrictions are imposed on the parameter values $\mu$. The integral representation of the Mittag-Leffler function represented in the following theorem will be called the representation " $\mathrm{B}$ ".

Theorem 3 (Representation "B"). For any real $\rho>1 / 2$ and any complex $\mu$ satisfying the condition $\Re \mu<$ $1+1 / \rho$ for the function $E_{\rho, \mu}(z)$, the following integral representations are valid:

1. for any real $\delta_{1 \rho}, \delta_{2 \rho}$ satisfying the conditions

$$
\begin{array}{lll}
\frac{\pi}{2 \rho}<\delta_{1 \rho} \leqslant \frac{\pi}{\rho}, & \frac{\pi}{2 \rho}<\delta_{2 \rho} \leqslant \frac{\pi}{\rho}, & \text { if } \rho>1, \\
\frac{\pi}{2 \rho}<\delta_{1 \rho}<\pi, & \frac{\pi}{2 \rho}<\delta_{2 \rho}<\pi, & \text { if } 1 / 2<\rho \leqslant 1,
\end{array}
$$

and any complex $z$ satisfying the condition $\frac{\pi}{2 \rho}-\delta_{2 \rho}+\pi<\arg z<-\frac{\pi}{2 \rho}+\delta_{1 \rho}+\pi$ the Mittag-Leffler function can be represented in the form

$$
E_{\rho, \mu}(z)=\int_{0}^{\infty} K_{\rho, \mu}\left(r,-\delta_{1 \rho}, \delta_{2 \rho}, z\right) d r,
$$

where $K_{\rho, \mu}\left(r, \varphi_{1}, \varphi_{2}, z\right)$ has the form (8);

2. if $1 / 2<\rho \leqslant 1$ and $\delta_{1 \rho}=\pi, \frac{\pi}{2 \rho}<\delta_{2 \rho}<\pi$, then for any complex $z$ satisfying the condition $\frac{\pi}{2 \rho}-\delta_{2 \rho}+\pi<$ $\arg z<-\frac{\pi}{2 \rho}+2 \pi$, the Mittag-Leffler function can be represented in the form

$$
\begin{aligned}
E_{\rho, \mu}(z)=\int_{0}^{\infty} K_{\rho, \mu}^{\prime}\left(r, \delta_{2 \rho}, z\right) d r-\int_{0}^{1-\varepsilon_{1}} K_{\rho, \mu}^{\prime}(r,-\pi, z) d r- & \\
& \int_{1+\varepsilon_{1}}^{\infty} K_{\rho, \mu}^{\prime}(r,-\pi, z) d r+\int_{-2 \pi}^{-\pi} P_{\rho, \mu}^{\prime}\left(\varepsilon_{1}, \psi,-2, z\right) d \psi,
\end{aligned}
$$

where $\varepsilon_{1}$ is an arbitrary real number satisfying the condition $0<\varepsilon_{1}<1$,

$$
K_{\rho, \mu}^{\prime}(r, \varphi, z)=\frac{\rho}{2 \pi i} \frac{\exp \left\{(z r)^{\rho} e^{-i \pi \rho} \cos (\rho \varphi)\right\}}{r^{2}+2 r \cos \varphi+1}(z r)^{\rho(1-\mu)} e^{i[\eta(r, \varphi, z)-\pi \rho(1-\mu)]}\left(r+e^{i \varphi}\right),
$$


where $\eta(r, \varphi, z)$ has the form (10) and

$$
\begin{aligned}
P_{\rho, \mu}^{\prime}(\tau, \psi, k, z)=\frac{\rho \tau}{2 \pi} \frac{\exp \left\{(z r(\tau, \psi))^{\rho} \cos (\rho \varphi(\tau, \psi, k))\right\}}{(r(\tau, \psi))^{2}-2 r(\tau, \psi) \cos (\varphi(\tau, \psi, k))+1} \times \\
\quad \times(z r(\tau, \psi))^{\rho(1-\mu)} e^{i\left[\chi^{\prime}(\tau, \psi, k, z)+\psi\right]}\left(r(\tau, \psi) e^{-i \varphi(\tau, \psi, k)}-1\right),
\end{aligned}
$$

where

$$
\begin{aligned}
\chi^{\prime}(\tau, \psi, k, z) & =(z r(\tau, \psi))^{\rho} \sin (\rho \varphi(\tau, \psi, k))+\rho(1-\mu) \varphi(\tau, \psi, k) \\
r(\tau, \psi) & =\sqrt{\tau^{2}+2 \tau \cos \psi+1} \\
\varphi(\tau, \psi, k) & =\arctan \left(\frac{\tau \sin \psi}{\tau \cos \psi+1}\right)+k \pi
\end{aligned}
$$

3. if $1 / 2<\rho \leqslant 1$ and $\frac{\pi}{2 \rho}<\delta_{1 \rho}<\pi, \delta_{2 \rho}=\pi$, then for any complex $z$ satisfying the condition $\frac{\pi}{2 \rho}<\arg z<$ $-\frac{\pi}{2 \rho}+\delta_{1 \rho}+\pi$, the Mittag-Leffler function can be represented in the form

$$
\begin{aligned}
E_{\rho, \mu}(z)=\int_{0}^{1-\varepsilon_{1}} K_{\rho, \mu}^{\prime}(r, \pi, z) d r-\int_{1+\varepsilon_{1}}^{\infty} K_{\rho, \mu}^{\prime}(r, \pi, z) d r+ & \\
& \quad+\int_{-\pi}^{0} P_{\rho, \mu}^{\prime}\left(\varepsilon_{1}, \psi, 0, z\right) d \psi-\int_{0}^{\infty} K_{\rho, \mu}^{\prime}\left(r,-\delta_{1 \rho}, z\right) d r,
\end{aligned}
$$

where $\varepsilon_{1}$ is an arbitrary real number satisfying the condition $0<\varepsilon_{1}<1$;

4. if $1 / 2<\rho \leqslant 1$ and $\delta_{1 \rho}=\delta_{2 \rho}=\pi$, then for any complex $z$ satisfying the condition $\frac{\pi}{2 \rho}<\arg z<-\frac{\pi}{2 \rho}+2 \pi$, the Mittag-Leffler function can be represented in the form

$$
\begin{aligned}
E_{\rho, \mu}(z)=\int_{0}^{1-\varepsilon_{1}} K_{\rho, \mu}(r, \pi, z) d r-\int_{1+\varepsilon_{1}}^{\infty} K_{\rho, \mu}(r, \pi, z) d r+ & \\
& +\int_{-\pi}^{0} P_{\rho, \mu}^{\prime}\left(\varepsilon_{1}, \psi, 0, z\right) d \psi+\int_{-2 \pi}^{-\pi} P_{\rho, \mu}^{\prime}\left(\varepsilon_{1}, \psi,-2, z\right) d \psi,
\end{aligned}
$$

where $\varepsilon_{1}$ is an arbitrary real number satisfying the condition $0<\varepsilon_{1}<1$ and $K_{\rho, \mu}(r, \delta, z)$ has the form (19).

Proof. The starting point of the proof is Theorem 1 and the integral representation (3) which is defined in it. In view of the notation (13), the representation (3) will be written in the form

$$
E_{\rho, \mu}(z)=\int_{\gamma_{\zeta}} \frac{\phi_{\rho, \mu}(\zeta, z)}{\zeta-1} d \zeta
$$

The problem consists in calculating this contour integral.

We consider an auxiliary integral

$$
I=\int_{\Gamma} \frac{\phi_{\rho, \mu}(\zeta, z)}{\zeta-1} d \zeta
$$

where the contour $\Gamma$ (see Figure 2) consists of the arc of the circle $C_{\epsilon}$ of radius $1+\epsilon$ with the center at the origin of coordinates, the segment $\Gamma_{2}$, the arc of the circle $C_{\varepsilon}$ the radius $\varepsilon$ with the center at the origin of coordinates and the segment $\Gamma_{1}$, which are defined in the following way: 


$$
\Gamma=\left\{\begin{array}{l}
C_{\epsilon}=\left\{\zeta:-\delta_{1 \rho}-\pi \leqslant \arg \zeta \leqslant \delta_{2 \rho}-\pi,|\zeta|=1+\epsilon\right\}, \\
\Gamma_{2}=\left\{\zeta: \arg \zeta=\delta_{2 \rho}-\pi, 1+\epsilon \geqslant|\zeta| \geqslant \varepsilon\right\}, \\
C_{\varepsilon}=\left\{\zeta: \delta_{2 \rho}-\pi \leqslant \arg \zeta \leqslant-\delta_{1 \rho}-\pi,|\zeta|=\varepsilon\right\}, \\
\Gamma_{1}=\left\{\zeta: \arg \zeta=-\delta_{1 \rho}-\pi, \varepsilon \leqslant|\zeta| \leqslant 1+\epsilon\right\} .
\end{array}\right.
$$

The contour is traversed in the positive direction. The cut of the complex plane $\zeta$ goes along the positive part of a real axis.

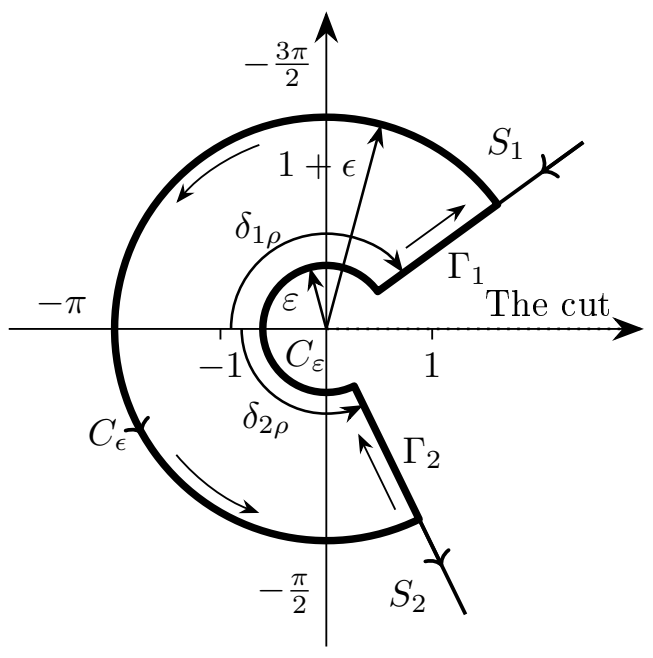

Figure 2. The auxiliary contour of integration $\Gamma$.

As it follows from Lemma 1, depending on a value of the parameter $\mu$, the integrand (38) has one or two poles. If $\Re \mu \geqslant 2$, then there are two poles in the points $\zeta=0$ and $\zeta=1$. If $\Re \mu \leqslant 1$, then there is one pole in the point $\zeta=1$. In addition, at non-integer values $\rho$ and $\mu$ the point $\zeta=0$ is the branch point. In the case when $\frac{1}{2}<\rho \leqslant 1$ and $\delta_{1 \rho}=\pi$ the segment $\Gamma_{1}$ will pass through a singular point $\zeta=1$ (Figure 2). The similar situation will be at $\frac{1}{2}<\rho \leqslant 1$ and $\delta_{2 \rho}=\pi$. In this case, the segment $\Gamma_{2}$ will pass through the point $\zeta=1$. These two cases will be considered separately. The case $\frac{1}{2}<\rho \leqslant 1, \delta_{1 \rho}=\delta_{2 \rho}=\pi$ also requires a separate consideration. In this case each of the segments $\Gamma_{1}$ and $\Gamma_{2}$ passes through a singular point $\zeta=1$. In view of the foregoing, it is necessary to consider four cases:

1.

$$
\begin{array}{lll}
\frac{\pi}{2 \rho}<\delta_{1 \rho} \leqslant \frac{\pi}{\rho}, & \frac{\pi}{2 \rho}<\delta_{2 \rho} \leqslant \frac{\pi}{\rho}, & \text { if } \quad \rho>1, \\
\frac{\pi}{2 \rho}<\delta_{1 \rho}<\pi, & \frac{\pi}{2 \rho}<\delta_{2 \rho}<\pi, & \text { if } \quad \frac{1}{2}<\rho \leqslant 1,
\end{array}
$$

2. $\frac{1}{2}<\rho \leqslant 1, \delta_{1 \rho}=\pi, \frac{\pi}{2 \rho}<\delta_{2 \rho}<\pi$,

3. $\quad \frac{1}{2}<\rho \leqslant 1, \delta_{2 \rho}=\pi, \frac{\pi}{2 \rho}<\delta_{1 \rho}<\pi$,

4. $\quad \frac{1}{2}<\rho \leqslant 1, \delta_{1 \rho}=\pi, \delta_{2 \rho}=\pi$.

Case 1 . We consider the first case at the beginning. Directly calculating the integral $I$ and substituting the variable of integration $\zeta=r e^{i \varphi}$ we obtain 


$$
\begin{aligned}
& I=\int_{C_{\epsilon}} \frac{\phi_{\rho, \mu}(\zeta, z)}{\zeta-1} d \zeta+\int_{\Gamma_{2}} \frac{\phi_{\rho, \mu}(\zeta, z)}{\zeta-1} d \zeta+\int_{C_{\varepsilon}} \frac{\phi_{\rho, \mu}(\zeta, z)}{\zeta-1} d \zeta+\int_{\Gamma_{1}} \frac{\phi_{\rho, \mu}(\zeta, z)}{\zeta-1} d \zeta= \\
& =\left.i \int_{-\delta_{1 \rho}-\pi}^{\delta_{2 \rho}-\pi} \frac{\phi_{\rho, \mu}\left(r e^{i \varphi}, z\right)}{r e^{i \varphi}-1} r e^{i \varphi} d \varphi\right|_{r=1+\epsilon}+\left.\int_{1+\varepsilon}^{\varepsilon} \frac{\phi_{\rho, \mu}\left(r e^{i \varphi}, z\right)}{r e^{i \varphi}-1} e^{i \varphi} d r\right|_{\varphi=\delta_{2 \rho}-\pi}+\left.i \int_{\delta_{2 \rho}-\pi}^{-\delta_{1 \rho}-\pi} \frac{\phi_{\rho, \mu}\left(r e^{i \varphi}, z\right)}{r e^{i \varphi}-1} r e^{i \varphi} d \varphi\right|_{r=\varepsilon} \\
& +\left.\int_{\varepsilon}^{1+\epsilon} \frac{\phi_{\rho, \mu}\left(r e^{i \varphi}, z\right)}{r e^{i \varphi}-1} e^{i \varphi} d r\right|_{\varphi=-\delta_{1 \rho}-\pi}=i \int_{-\delta_{1 \rho}-\pi}^{\delta_{2 \rho}-\pi} \frac{\phi_{\rho, \mu}\left((1+\epsilon) e^{i \varphi}, z\right)}{(1+\epsilon) e^{i \varphi}-1}(1+\epsilon) e^{i \varphi} d \varphi+\int_{1+\epsilon}^{\varepsilon} \frac{\phi_{\rho, \mu}\left(r e^{i\left(\delta_{2 \rho}-\pi\right)}, z\right)}{r e^{i\left(\delta_{2 \rho}-\pi\right)}-1} e^{i\left(\delta_{2 \rho}-\pi\right)} d r \\
& +i \int_{\delta_{2 \rho}-\pi}^{-\delta_{1 \rho}-\pi} \frac{\phi_{\rho, \mu}\left(\varepsilon e^{i \varphi}, z\right)}{\varepsilon e^{i \varphi}-1} \varepsilon e^{i \varphi} d \varphi+\int_{\varepsilon}^{1+\epsilon} \frac{\phi_{\rho, \mu}\left(r e^{i\left(-\delta_{1 \rho}-\pi\right)}, z\right)}{r e^{i\left(-\delta_{1 \rho}-\pi\right)}-1} e^{i\left(-\delta_{1 \rho}-\pi\right)} d r=I_{C_{\epsilon}}+I_{\Gamma_{2}}+I_{C_{\varepsilon}}+I_{\Gamma_{1}} .
\end{aligned}
$$

Now we let $\varepsilon \rightarrow 0$ in this expression and we will study the behavior of $I_{C_{\varepsilon}}, I_{\Gamma_{1}}$ and $I_{\Gamma_{2}}$. We consider the integral $I_{C_{\varepsilon}}$ at the beginning. For this integral the relation is true

$$
\lim _{\varepsilon \rightarrow 0}\left|I_{C_{\varepsilon}}\right| \leqslant \lim _{\varepsilon \rightarrow 0} \int_{\delta_{2 \rho}-\pi}^{-\delta_{1 \rho}-\pi}\left|\frac{\phi_{\rho, \mu}\left(\varepsilon e^{i \varphi}, z\right)}{\varepsilon e^{i \varphi}-1} \varepsilon e^{i \varphi}\right||d \varphi| .
$$

We consider the integrand. To get rid of the complexity in the denominator we multiply and divide the integrand by $\varepsilon e^{-i \varphi}-1$. We also represent $z=|z| e^{i \arg z}$ and $\mu=\mu_{R}+i \mu_{I}$, and for a power function we will use the representation $\xi^{a}=\exp \{a \ln \xi\}, \xi>0$. As a result, we obtain

$$
\begin{gathered}
\lim _{\varepsilon \rightarrow 0}\left|\frac{\phi_{\rho, \mu}\left(\varepsilon e^{i \varphi}, z\right)}{\varepsilon e^{i \varphi}-1} \varepsilon e^{i \varphi}\right|=\frac{\rho}{2 \pi} \lim _{\varepsilon \rightarrow 0}\left|\frac{\exp \left\{\left(z \varepsilon e^{i \varphi}\right)^{\rho}\right\}\left(z \varepsilon e^{i \varphi}\right)^{\rho(1-\mu)} \varepsilon e^{i \varphi}\left(\varepsilon e^{-i \varphi}-1\right)}{\left(\varepsilon e^{i \varphi}-1\right)\left(\varepsilon e^{-i \varphi}-1\right)}\right| \\
=\frac{\rho}{2 \pi} \lim _{\varepsilon \rightarrow 0}\left|\frac{\exp \left\{(|z| \varepsilon)^{\rho} e^{i \rho(\varphi+\arg z)}\right\}(|z| \varepsilon)^{\rho\left(1-\mu_{R}-i \mu_{I}\right)} e^{i \rho\left(1-\mu_{R}-i \mu_{I}\right)(\varphi+\arg z)} \varepsilon\left(\varepsilon-e^{i \varphi}\right)}{\varepsilon^{2}-2 \varepsilon \cos \varphi+1}\right| \\
=\frac{\rho}{2 \pi} \lim _{\varepsilon \rightarrow 0} \mid \frac{1}{\varepsilon^{2}-2 \varepsilon \cos \varphi+1} \exp \left\{(|z| \varepsilon)^{\rho} e^{i \rho(\varphi+\arg z)}+\rho\left(1-\mu_{R}-i \mu_{I}\right) \ln (|z| \varepsilon)+\right. \\
\left.+i \rho\left(1-\mu_{R}-i \mu_{I}\right)(\varphi+\arg z)+\ln \varepsilon\right\}\left(\varepsilon-e^{i \varphi}\right) \mid \\
=\frac{\rho}{2 \pi} \lim _{\varepsilon \rightarrow 0} \frac{1}{\varepsilon^{2}-2 \varepsilon \cos \varphi+1} \mid \exp \left\{(|z| \varepsilon)^{\rho} \cos (\rho(\varphi+\arg z))+\rho\left(1-\mu_{R}\right) \ln (|z| \varepsilon)+\rho(\varphi+\arg z) \mu_{I}+\ln \varepsilon\right. \\
\left.+i\left[(|z| \varepsilon)^{\rho} \sin (\rho(\varphi+\arg z))+\rho\left(1-\mu_{R}\right)(\varphi+\arg z)-\rho \ln (|z| \varepsilon) \mu_{I}\right]\right\}\left(\varepsilon-e^{-i \varphi}\right) \mid \\
=\frac{\rho}{2 \pi} \lim _{\varepsilon \rightarrow 0} \frac{\left|e^{A+i B}\left(\varepsilon-e^{i \varphi}\right)\right|}{\varepsilon^{2}-2 \varepsilon \cos \varphi+1}
\end{gathered}
$$

where

$$
\begin{aligned}
& A=(|z| \varepsilon)^{\rho} \cos (\rho(\varphi+\arg z))+\rho\left(1-\mu_{R}\right) \ln (|z| \varepsilon)+\rho(\varphi+\arg z) \mu_{I}+\ln \varepsilon \\
& B=(|z| \varepsilon)^{\rho} \sin (\rho(\varphi+\arg z))+\rho\left(1-\mu_{R}\right)(\varphi+\arg z)-\rho \mu_{I} \ln (|z| \varepsilon) .
\end{aligned}
$$


For the numerator we have the estimate

$$
\left|e^{A+i B}\left(\varepsilon-e^{i \varphi}\right)\right| \leqslant\left|e^{A+i B} \varepsilon\right|+\left|e^{A+i B+i \varphi}\right|=e^{A}(\varepsilon+1) .
$$

Thus,

$$
\begin{aligned}
& \frac{\rho}{2 \pi} \lim _{\varepsilon \rightarrow 0} \frac{\left|e^{A+i B}\left(\varepsilon-e^{i \varphi}\right)\right|}{\varepsilon^{2}-2 \varepsilon \cos \varphi+1} \leqslant \frac{\rho}{2 \pi} \lim _{\varepsilon \rightarrow 0} \frac{e^{A}(\varepsilon+1)}{\varepsilon^{2}-2 \varepsilon \cos \varphi+1} \\
& =\frac{\rho}{2 \pi} \lim _{\varepsilon \rightarrow 0} \frac{1}{\varepsilon^{2}-2 \varepsilon \cos \varphi+1} \exp \left\{(|z| \varepsilon)^{\rho} \cos (\rho(\varphi+\arg z))\right. \\
& \left.+\rho\left(1-\mu_{R}\right) \ln (|z| \varepsilon)+\rho(\varphi+\arg z) \mu_{I}+\ln \varepsilon+\ln (\varepsilon+1)\right\} \\
& =\frac{\rho}{2 \pi} \lim _{\varepsilon \rightarrow 0} \frac{1}{\varepsilon^{2}-2 \varepsilon \cos \varphi+1} \exp \left\{(|z| \varepsilon)^{\rho} \cos (\rho(\varphi+\arg z))+\right. \\
& \left.+\left(\rho\left(1-\mu_{R}\right)+1\right) \ln \varepsilon+\ln (\varepsilon+1)+\rho\left(1-\mu_{R}\right) \ln (|z|)+\rho \mu_{I}(\varphi+\arg z)\right\} \\
& =\left\{\begin{array}{cl}
0, & \mu_{R}<1+\frac{1}{\rho}, \\
\frac{\rho}{2 \pi|z|} e^{\rho(\varphi+\arg z) \mu_{I},}, & \mu_{R}=1+\frac{1}{\rho} .
\end{array}\right.
\end{aligned}
$$

From this it follows that

$$
\lim _{\varepsilon \rightarrow 0} I_{C_{\varepsilon}}=0, \quad \text { if } \quad \mu_{R}<1+1 / \rho .
$$

Now we consider the behavior of the integral $I_{\Gamma_{1}}$ at $\varepsilon \rightarrow 0$. We have the estimate

$$
\begin{array}{r}
\lim _{\varepsilon \rightarrow 0}\left|\int_{\varepsilon}^{1+\epsilon} \frac{\phi_{\rho, \mu}\left(r e^{i\left(-\delta_{1 \rho}-\pi\right)}, z\right)}{r e^{i\left(-\delta_{1 \rho}-\pi\right)}-1} e^{i\left(-\delta_{1 \rho}-\pi\right)} d r\right| \leqslant \lim _{\varepsilon \rightarrow 0} \int_{\varepsilon}^{1+\epsilon}\left|\frac{\phi_{\rho, \mu}\left(r e^{i\left(-\delta_{1 \rho}-\pi\right)}, z\right)}{r e^{i\left(-\delta_{1 \rho}-\pi\right)}-1} e^{i\left(-\delta_{1 \rho}-\pi\right)}\right||d r| \\
=\int_{0}^{1+\epsilon}\left|\frac{\phi_{\rho, \mu}\left(r e^{i\left(-\delta_{1 \rho}-\pi\right)}, z\right)}{r e^{i\left(-\delta_{1 \rho}-\pi\right)}-1} e^{i\left(-\delta_{1 \rho}-\pi\right)}\right||d r| .
\end{array}
$$

Consequently, it is necessary to study the behavior of the integrand at $r \rightarrow 0$. For convenience, we introduce the notation $\varphi_{1}=-\delta_{1 \rho}-\pi$. Further, similarly to the previous case, we get rid of the complexity in the denominator. To do this, we multiply and divide the integrand by the complex conjugate value of the denominator, i.e., by $\left(r e^{-i \varphi_{1}}-1\right)$ and represent $z=|z| e^{i \arg z}, \mu=\mu_{R}+\mu_{I}$. For a power function we will make use of the representation $\xi^{a}=\exp \{a \ln \xi\}$. Using (13) we get

$$
\begin{array}{r}
\lim _{r \rightarrow 0}\left|\frac{\phi_{\rho, \mu}\left(r e^{i\left(-\delta_{1 \rho}-\pi\right)}, z\right)}{r e^{i\left(-\delta_{1 \rho}-\pi\right)}-1} e^{i\left(-\delta_{1 \rho}-\pi\right)}\right|=\frac{\rho}{2 \pi} \lim _{r \rightarrow 0}\left|\frac{\exp \left\{\left(z r e^{i \varphi_{1}}\right)^{\rho}\right\}\left(z r e^{i \varphi_{1}}\right)^{\rho(1-\mu)} e^{i \varphi_{1}}\left(r e^{-i \varphi_{1}}-1\right)}{\left(r e^{i \varphi_{1}}-1\right)\left(r e^{-i \varphi_{1}}-1\right)}\right| \\
=\frac{\rho}{2 \pi} \lim _{r \rightarrow 0}\left|\frac{1}{r^{2}-2 r \cos \varphi_{1}+1} \exp \left\{(|z| r)^{\rho} e^{i \rho\left(\varphi_{1}+\arg z\right)}\right\}(|z| r)^{\rho\left(1-\mu_{R}+i \mu_{I}\right)} e^{i \rho\left(1-\mu_{R}-i \mu_{I}\right)\left(\varphi_{1}+\arg z\right)}\left(r-e^{i \varphi_{1}}\right)\right| \\
=\frac{\rho}{2 \pi} \lim _{r \rightarrow 0} \frac{1}{r^{2}-2 r \cos \varphi_{1}+1} \mid \exp \left\{(|z| r)^{\rho} \cos \left(\rho\left(\varphi_{1}+\arg z\right)\right)+\rho \mu_{I}\left(\varphi_{1}+\arg z\right)+\rho\left(1-\mu_{R}\right) \ln (|z| r)\right. \\
\left.+i\left[(|z| r)^{\rho} \sin \left(\rho\left(\varphi_{1}+\arg z\right)\right)+\rho\left(1-\mu_{R}\right)\left(\varphi_{1}+\arg z\right)-\rho \mu_{I} \ln (|z| r)\right]\right\}\left(r-e^{i \varphi_{1}}\right) \mid \\
=\frac{\rho}{2 \pi} \lim _{r \rightarrow 0} \frac{\left|e^{A_{1}+i B_{1}}\left(r-e^{i \varphi_{1}}\right)\right|}{r^{2}-2 r \cos \varphi_{1}+1},
\end{array}
$$


where

$$
\begin{aligned}
A_{1} & =(|z| r)^{\rho} \cos \left(\rho\left(\varphi_{1}+\arg z\right)\right)+\rho \mu_{I}\left(\varphi_{1}+\arg z\right)+\rho\left(1-\mu_{R}\right) \ln (|z| r), \\
B_{1} & =(|z| r)^{\rho} \sin \left(\rho\left(\varphi_{1}+\arg z\right)\right)+\rho\left(1-\mu_{R}\right)\left(\varphi_{1}+\arg z\right)-\rho \mu_{I} \ln (|z| r) .
\end{aligned}
$$

For the numerator we have the estimate

$$
\left|e^{A_{1}+i B_{1}}\left(r-e^{i \varphi_{1}}\right)\right| \leqslant\left|r e^{A_{1}+i B_{1}}\right|+\left|e^{A_{1}+i B_{1}+i \varphi_{1}}\right|=e^{A_{1}}(r+1) .
$$

Consequently, we obtain

$$
\begin{aligned}
& \frac{\rho}{2 \pi} \lim _{r \rightarrow 0} \frac{\left|e^{A_{1}+i B_{1}}\left(r-e^{i \varphi_{1}}\right)\right|}{r^{2}-2 r \cos \varphi_{1}+1} \leqslant \frac{\rho}{2 \pi} \lim _{r \rightarrow 0} \frac{e^{A_{1}}(r+1)}{r^{2}-2 r \cos \varphi_{1}+1} \\
& =\frac{\rho}{2 \pi} \lim _{r \rightarrow 0} \frac{1}{r^{2}-2 r \cos \varphi_{1}+1} \exp \left\{(|z| r)^{\rho} \cos \left(\rho\left(\varphi_{1}+\arg z\right)\right)+\rho \mu_{I}\left(\varphi_{1}+\arg z\right)\right. \\
& \left.+\rho\left(1-\mu_{R}\right) \ln (|z| r)+\ln (r+1)\right\}=\left\{\begin{array}{cc}
0, & \mu_{R}<1, \\
\frac{\rho}{2 \pi} e^{\rho\left(\varphi_{1}+\arg z\right) \mu_{I}}, & \mu_{R}=1 .
\end{array}\right.
\end{aligned}
$$

We get the similar result for the integral $I_{\Gamma_{2}}$

$$
\lim _{\varepsilon \rightarrow 0}\left|\frac{\phi_{\rho, \mu}\left(r e^{i\left(\delta_{2 \rho}-\pi\right)}, z\right)}{r e^{i\left(\delta_{2 \rho}-\pi\right)}-1} e^{i\left(\delta_{2 \rho}-\pi\right)}\right|=\left\{\begin{array}{cl}
0, & \mu_{R}<1, \\
\frac{\rho}{2 \pi} e^{\rho\left(\delta_{2 \rho}-\pi+\arg z\right) \Im \mu}, & \mu_{R}=1 .
\end{array}\right.
$$

Thus, at $\Re \mu \leqslant 1$ the limits $\lim _{\mathcal{E} \rightarrow 0} I_{\Gamma_{1}}$ and $\lim _{\mathcal{E} \rightarrow 0} I_{\Gamma_{2}}$ will converge to the corresponding definite integrals

$$
\begin{aligned}
& \lim _{\varepsilon \rightarrow 0} I_{\Gamma_{1}}=\int_{0}^{1+\epsilon} \frac{\phi_{\rho, \mu}\left(r e^{i\left(-\delta_{1 \rho}-\pi\right)}, z\right)}{r e^{i\left(-\delta_{1 \rho}-\pi\right)}-1} e^{i\left(-\delta_{1 \rho}-\pi\right)} d r, \\
& \lim _{\varepsilon \rightarrow 0} I_{\Gamma_{2}}=-\int_{0}^{1+\epsilon} \frac{\phi_{\rho, \mu}\left(r e^{i\left(\delta_{2 \rho}-\pi\right)}, z\right)}{r e^{i\left(\delta_{2 \rho}-\pi\right)}-1} e^{i\left(\delta_{2 \rho}-\pi\right)} d r .
\end{aligned}
$$

We will pay attention to the fact that in the case under consideration when the parameters $\rho, \delta_{1 \rho}, \delta_{2 \rho}$ satisfy the condition (40), the integrand of the integral $I$ (see (38)) inside the region limited by the contour $\Gamma$, is an analytical function. Consequently, from (41) we have

$$
I=I_{C_{\epsilon}}+I_{\Gamma_{2}}+I_{C_{\varepsilon}}+I_{\Gamma_{1}}=0
$$

Letting $\varepsilon \rightarrow 0$ in this expression and taking into consideration (42) we obtain

$$
I_{C_{e}}=-\lim _{\varepsilon \rightarrow 0}\left(I_{\Gamma_{1}}+I_{\Gamma_{2}}\right) .
$$

Substituting here the expression for $I_{C_{\epsilon}}$ and using (43) we obtain 


$$
\begin{aligned}
i \int_{-\delta_{1 \rho}-\pi}^{\delta_{2 \rho}-\pi} & \frac{\phi_{\rho, \mu}\left((1+\epsilon) e^{i \varphi}, z\right)}{(1+\epsilon) e^{i \varphi}-1}(1+\epsilon) e^{i \varphi} d \varphi \\
& =-\int_{0}^{1+\epsilon} \frac{\phi_{\rho, \mu}\left(r e^{i\left(-\delta_{1 \rho}-\pi\right)}, z\right)}{r e^{i\left(-\delta_{1 \rho}-\pi\right)}-1} e^{i\left(-\delta_{1 \rho}-\pi\right)} d r+\int_{0}^{1+\epsilon} \frac{\phi_{\rho, \mu}\left(r e^{i\left(\delta_{2 \rho}-\pi\right)}, z\right)}{r e^{i\left(\delta_{2 \rho}-\pi\right)}-1} e^{i\left(\delta_{2 \rho}-\pi\right)} d r, \quad \mu_{R}<1+\frac{1}{\rho} .
\end{aligned}
$$

Here it should be pointed out that at values $1<\mu_{R}<1+1 / \rho$ the integrals (43) have a singularity in the point $r=0$ and at values $\mu_{R} \leqslant 1$ the singularity disappears.

Now let us return to the Mittag-Leffler function. Directly calculating (37) and replacing the variable of integration $\zeta=r e^{i \varphi}$ we get

$$
\begin{array}{r}
E_{\rho, \mu}(z)=\int_{S_{1}} \frac{\phi_{\rho, \mu}(\zeta, z)}{\zeta-1} d \zeta+\int_{C_{e}} \frac{\phi_{\rho, \mu}(\zeta, z)}{\zeta-1} d \zeta+\int_{S_{2}} \frac{\phi_{\rho, \mu}(\zeta, z)}{\zeta-1} d \zeta=\left.\int_{\infty}^{1+\epsilon} \frac{\phi_{\rho, \mu}\left(r e^{i \varphi}, z\right)}{r e^{i \varphi}-1} e^{i \varphi} d r\right|_{\varphi=-\delta_{1 \rho}-\pi} \\
+\left.i \int_{-\delta_{1 \rho}-\pi}^{\delta_{2 \rho}-\pi} \frac{\phi_{\rho, \mu}\left(r e^{i \varphi}, z\right)}{r e^{i \varphi}-1} r e^{i \varphi} \varphi\right|_{r=1+\epsilon} \quad+\left.\int_{1+\epsilon}^{\infty} \frac{\phi_{\rho, \mu}\left(r e^{i \varphi}, z\right)}{r e^{i \varphi}-1} e^{i \varphi} d r\right|_{\varphi=\delta_{2 \rho}-\pi}=\int_{\infty}^{1+\epsilon} \frac{\phi_{\rho, \mu}\left(r e^{i\left(-\delta_{1 \rho}-\pi\right)}, z\right)}{r e^{i\left(-\delta_{1 \rho}-\pi\right)}-1} e^{i\left(-\delta_{1 \rho}-\pi\right)} d r \\
+i \int_{-\delta_{1 \rho}-\pi}^{\delta_{2 \rho}-\pi} \frac{\phi_{\rho, \mu}\left((1+\epsilon) e^{i \varphi}, z\right)}{(1+\epsilon) e^{i \varphi}-1}(1+\epsilon) e^{i \varphi} \varphi+\int_{1+\epsilon}^{\infty} \frac{\phi_{\rho, \mu}\left(r e^{i\left(\delta_{2 \rho}-\pi\right)}, z\right)}{r e^{i\left(\delta_{2 \rho}-\pi\right)}-1} e^{i\left(\delta_{2 \rho}-\pi\right)} d r .
\end{array}
$$

Using here (45) we obtain

$$
E_{\rho, \mu}(z)=\int_{0}^{\infty}\left(\frac{\phi_{\rho, \mu}\left(r e^{i\left(\delta_{2 \rho}-\pi\right)}, z\right)}{r e^{i\left(\delta_{2 \rho}-\pi\right)}-1} e^{i\left(\delta_{2 \rho}-\pi\right)}-\frac{\phi_{\rho, \mu}\left(r e^{i\left(-\delta_{1 \rho}-\pi\right)}, z\right)}{r e^{i\left(-\delta_{1 \rho}-\pi\right)}-1} e^{i\left(-\delta_{1 \rho}-\pi\right)}\right) d r, \quad \mu_{R}<1+\frac{1}{\rho} .
$$

We consider the first summand in this expression. Getting rid of the complexity in the denominator and using (13) we get

$$
\begin{gathered}
\frac{\phi_{\rho, \mu}\left(r e^{i\left(\delta_{2 \rho}-\pi\right)}, z\right)}{r e^{i\left(\delta_{2 \rho}-\pi\right)}-1} e^{i\left(\delta_{2 \rho}-\pi\right)}=\frac{\phi_{\rho, \mu}\left(r e^{i\left(\delta_{2 \rho}-\pi\right)}, z\right) e^{i\left(\delta_{2 \rho}-\pi\right)}\left(r e^{-i\left(\delta_{2 \rho}-\pi\right)}-1\right)}{\left.\left(r e^{i\left(\delta_{2 \rho}-\pi\right)}-1\right)\right)\left(r e^{-i\left(\delta_{2 \rho}-\pi\right)}-1\right)} \\
=\frac{\phi_{\rho, \mu}\left(r e^{i\left(\delta_{2 \rho}-\pi\right)}, z\right)\left(r-e^{i\left(\delta_{2 \rho}-\pi\right)}\right)}{\left.r^{2}+2 r \cos \delta_{2 \rho}+1\right)}=\frac{\rho}{2 \pi i} \frac{\exp \left\{\left(z r e^{i\left(\delta_{2 \rho}-\pi\right)}\right)^{\rho}\right\}\left(z r e^{i\left(\delta_{2 \rho}-\pi\right)}\right)^{\rho(1-\mu)}\left(r+e^{i \delta_{2 \rho}}\right)}{r^{2}+2 r \cos \delta_{2 \rho}+1} \\
=\frac{\rho}{2 \pi i} \frac{\exp \left\{(z r)^{\rho} e^{-i \rho \pi}\left(\cos \left(\rho \delta_{2 \rho}\right)+i \sin \left(\rho \delta_{2 \rho}\right)\right)\right\}(z r)^{\rho(1-\mu)} e^{i \rho(1-\mu)\left(\delta_{2 \rho}-\pi\right)}\left(r+e^{i \delta_{2 \rho}}\right)}{r^{2}+2 r \cos \delta_{2 \rho}+1} \\
=\frac{\rho}{2 \pi i} \frac{\exp \left\{(z r)^{\rho} e^{-i \rho \pi} \cos \left(\rho \delta_{2 \rho}\right)\right\}(z r)^{\rho(1-\mu)} e^{-i \rho(1-\mu) \pi} e^{i \eta\left(r, \delta_{2 \rho}, z\right)}\left(r+e^{i \delta_{2 \rho}}\right)}{r^{2}+2 r \cos \delta_{2 \rho}+1}
\end{gathered}
$$


where $\eta(r, \varphi, z)$ has the form (10). Similarly, for the second summand in (46) we have

$$
\begin{aligned}
\frac{\phi_{\rho, \mu}\left(r e^{i\left(-\delta_{2 \rho}-\pi\right)}, z\right)}{r e^{i\left(-\delta_{2 \rho}-\pi\right)}-1} e^{i\left(-\delta_{2 \rho}-\pi\right)}= & \\
& =\frac{\rho}{2 \pi i} \frac{\exp \left\{(z r)^{\rho} e^{-i \rho \pi} \cos \left(\rho \delta_{1 \rho}\right)\right\}(z r)^{\rho(1-\mu)} e^{-i \rho(1-\mu) \pi} e^{i \eta\left(r,-\delta_{1 \rho} z\right)}\left(r+e^{-i \delta_{1 \rho}}\right)}{r^{2}+2 r \cos \delta_{1 \rho}+1}
\end{aligned}
$$

Now substituting (47) and (48) in (46) and making simple transformations we finally obtain

$$
E_{\rho, \mu}(z)=\int_{0}^{\infty} K_{\rho, \mu}\left(r,-\delta_{1 \rho}, \delta_{2 \rho}, z\right) d r
$$

where $K_{\rho, \mu}\left(r, \varphi_{1}, \varphi_{2}, z\right)$ is defined by the expression (8). The values $\delta_{1 \rho}$ and $\delta_{2 \rho}$ satisfy the conditions (40). The condition for the value $\arg z$ did not change and passes from Theorem 1 without any changes. Thus, the first item of the theorem is proved.

Case 2. Now we consider the case when $\frac{1}{2}<\rho \leqslant 1, \delta_{1 \rho}=\pi$ and $\pi /(2 \rho)<\delta_{2 \rho}<\pi$. As it was mentioned earlier, in this case the segment $\Gamma_{1}$ of the auxiliary contour $\Gamma$ (see Figure 2) will go through the singular point $\zeta=1$. That is why, it is necessary to change the contour of integration in such a way that one could bypass this point leaving it outside the contour. The auxiliary contour $\Gamma^{\prime}$ that we get will consist of the arc of the circle $C_{\epsilon}$ with the center at the origin of coordinates and radius $1+\epsilon, \epsilon>0$ (see Figure 3), the segment $A B$, the circle $C_{\varepsilon}$ with the center at the origin of coordinates and radius $\varepsilon$ satisfying the condition $0<\varepsilon<1$ ), the segment $C D$, the arc of the circle $C_{1 \varepsilon_{1}}^{\prime}$ with the center in the point $\zeta^{\prime}=1\left(\arg \zeta^{\prime}=-2 \pi\right)$ and radius $\varepsilon_{1}$ (here $\varepsilon_{1}<1-\varepsilon$ and $\left.\varepsilon_{1}<\epsilon\right)$ and the segment $E F$.

For further study we need to parametrize this contour. As a result, in the plane $\zeta$ the arc of the circle $C_{\epsilon}$ can be written in the form $C_{\epsilon}=\left\{\zeta:-2 \pi \leqslant \arg \zeta \leqslant \delta_{2 \rho}-\pi,|\zeta|=1+\epsilon\right\}$. The segment $A B$ is written in the form $A B=\left\{\zeta: \arg \zeta=\delta_{2 \rho}-\pi, \varepsilon \leqslant|\zeta| \leqslant 1+\epsilon\right\}$. The arc of the circle $C_{\varepsilon}$ has the form $C_{\varepsilon}=\left\{\zeta: \delta_{2 \rho}-\pi \leqslant \arg \zeta \leqslant-2 \pi,|\zeta|=\varepsilon\right\}$. The segments $C D$ and $E F$ are written in the form $C D=\left\{\zeta: \arg \zeta=-2 \pi, \varepsilon \leqslant|\zeta| \leqslant 1-\varepsilon_{1}\right\}$ and $E F=\left\{\zeta: \arg \zeta=-2 \pi, 1+\varepsilon_{1} \leqslant|\zeta| \leqslant 1+\epsilon\right\}$.

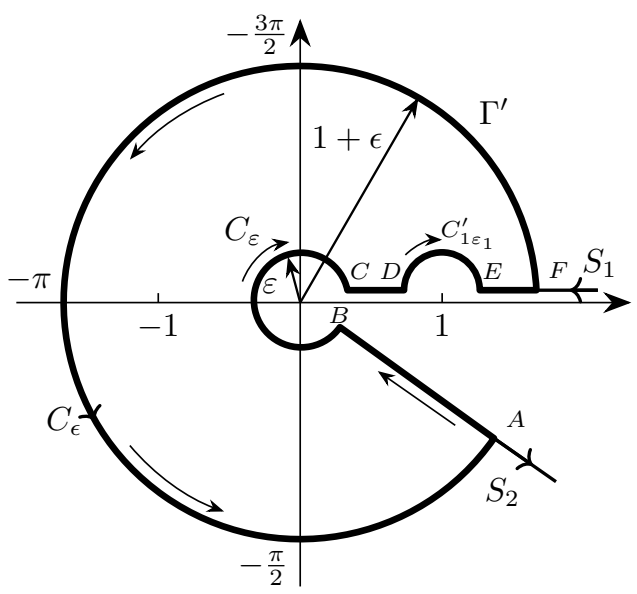

Figure 3. The auxiliary contour of integration $\Gamma^{\prime}$.

To parametrize the arc of the circle $C_{1 \varepsilon_{1}}^{\prime}$, we consider the mapping $u=\zeta-1$ of the complex plane $\zeta$ on the complex plane $u$. This mapping is a conformal mapping and is a left shift of the entire complex plane $\zeta$ by the value 1 . As a result of such a shift, the point $\zeta=1$ in the plane $\zeta$ is mapped onto the point $u=0$ of the plane $u$. Thus, the circle $C_{1 \varepsilon_{1}}^{\prime}$ with the center in the point $\zeta=1$ and the radius $\varepsilon_{1}<1$ of the 
plane $\zeta$ will be mapped on the circle $C_{0 \varepsilon_{1}}^{u}$ with the center in the point $u=0$ and the radius $\varepsilon_{1}$ on the plane $u$. Thus, to parametrize the arc of the circle $C_{1 \varepsilon_{1}}^{\prime}$ in the plane $\zeta$ it is enough to parametrize the arc of the circle $C_{0 \varepsilon_{1}}^{u}$ in the complex plane $u$ and then to map the complex plane $u$ on to the plane $\zeta$ with the help of an inverse conformal mapping $\zeta=u+1$.

We will represent the complex number $u$ in the form $u=\tau e^{i \psi}$. Then, an arc of a circle $C_{0 \varepsilon_{1}}^{u}$ in the complex plane $u$ has the form $C_{0 \varepsilon_{1}}^{u}=\left\{u:-2 \pi \leqslant \psi \leqslant-\pi, \tau=\varepsilon_{1}\right\}$, where $\varepsilon_{1} \leqslant 1$. Now we map the complex plane $u$ onto the plane $\zeta$. We have $\zeta=\tau e^{i \psi}+1=\tau \cos \psi+i \tau \sin \psi+1$. From this we obtain

$$
|\zeta|=\sqrt{\tau^{2}+2 \tau \cos \psi+1}, \quad \arg \zeta=\arctan \left(\frac{\tau \sin \psi}{\tau \cos \psi+1}\right) \text {. }
$$

Here it is necessary to note that $\arctan (x)$ is a multivalued function. The principal branch of this function takes values in the interval $[-\pi / 2, \pi / 2]$. However, as one can see from the definition of the auxiliary contour $\Gamma^{\prime}$, the center of the arc of the circle $C_{1 \varepsilon_{1}}^{\prime}$ lies in the point $\zeta^{\prime}=1$ in which $\arg \zeta^{\prime}=-2 \pi$. Therefore, it is necessary to choose the required branch in $\arctan (x)$ in such a way that the mapping (49) could map an arc of a circle $C_{0 \varepsilon_{1}}^{u}$ of the plane $u$ on to an arc of a circle $C_{1 \varepsilon_{1}}^{\prime}$ of the plane $\zeta$ with the center in the point $\zeta^{\prime}=1$ and $\arg \zeta^{\prime}=-2 \pi$. As a result, the mapping (49) will take the form

$$
|\zeta|=\sqrt{\tau^{2}+2 \tau \cos \psi+1}, \quad \arg \zeta=\arctan \left(\frac{\tau \sin \psi}{\tau \cos \psi+1}\right)+k \pi
$$

For the arc of the circle $C_{1 \varepsilon_{1}}^{\prime}$ we obtain $k=-2$. Here it should be pointed out that these formulas produce the mapping of the circle $C_{0 \varepsilon_{1}}^{u}$ on to the circle $C_{1 \varepsilon_{1}}^{\prime}$ only in the case $\tau \leqslant 1$. If $\tau>1$, then these formulas will not be true.

Thus, in view of the remarks made the auxiliary contour $\Gamma^{\prime}$ can be represented in the form

$$
\Gamma^{\prime}=\left\{\begin{array}{l}
C_{\epsilon}=\left\{\zeta:-2 \pi \leqslant \arg \zeta \leqslant \delta_{2 \rho}-\pi,|\zeta|=1+\epsilon\right\}, \\
A B=\left\{\zeta: \arg \zeta=\delta_{2 \rho}-\pi, \varepsilon \leqslant|\zeta| \leqslant 1+\epsilon\right\}, \\
C_{\varepsilon}=\left\{\zeta: \delta_{2 \rho}-\pi \leqslant \arg \zeta \leqslant-2 \pi,|\zeta|=\varepsilon\right\}, \\
C D=\left\{\zeta: \arg \zeta=-2 \pi, \varepsilon \leqslant|\zeta| \leqslant 1-\varepsilon_{1}\right\}, \\
C_{1 \varepsilon_{1}}^{\prime}=\left\{\begin{array}{c}
\arg \zeta=\arctan \left(\frac{\tau \sin \psi}{\tau \cos \psi+1}\right)+k \pi, \\
\zeta: \quad \begin{array}{c}
|\zeta|=\sqrt{\tau^{2}+2 \tau \cos \psi+1} \\
-\pi \geqslant \psi \geqslant-2 \pi, \tau=\varepsilon_{1}, k=-2,
\end{array} \\
E F=\left\{\zeta: \arg \zeta=-2 \pi, 1+\varepsilon_{1} \leqslant|\zeta| \leqslant 1+\epsilon\right\} .
\end{array}\right\}
\end{array}\right.
$$

The contour is traversed in a positive direction.

Next, we consider an auxiliary integral

$$
I^{\prime}=\int_{\Gamma^{\prime}} \frac{\phi_{\rho, \mu}(\zeta, z)}{\zeta-1} d \zeta
$$

By calculating this integral we have

$$
\begin{aligned}
I^{\prime}=\int_{\Gamma^{\prime}} \frac{\phi_{\rho, \mu}(\zeta, z)}{\zeta-1} d \zeta= & \int_{C_{\varepsilon}} \frac{\phi_{\rho, \mu}(\zeta, z)}{\zeta-1} d \zeta+\int_{A B} \frac{\phi_{\rho, \mu}(\zeta, z)}{\zeta-1} d \zeta+\int_{C_{\varepsilon}} \frac{\phi_{\rho, \mu}(\zeta, z)}{\zeta-1} d \zeta+\int_{C D} \frac{\phi_{\rho, \mu}(\zeta, z)}{\zeta-1} d \zeta+ \\
& \int_{C_{1 \varepsilon_{1}}^{\prime}} \frac{\phi_{\rho, \mu}(\zeta, z)}{\zeta-1} d \zeta+\int_{E F} \frac{\phi_{\rho, \mu}(\zeta, z)}{\zeta-1} d \zeta=I_{C_{\varepsilon}}+I_{A B}^{\prime}+I_{C_{\varepsilon}}+I_{C D}^{\prime}+I_{C_{1 \varepsilon_{1}}^{\prime}}^{\prime}+I_{E F}^{\prime} .
\end{aligned}
$$


One should pay attention to the fact that inside the region limited by the contour $\Gamma^{\prime}$ the integrand $\phi_{\rho, \mu}(\zeta, z) /(\zeta-1)$ of the integral $I^{\prime}$ is an analytical function of the complex variable $\zeta$. Consequently, according to the Cauchy integral theorem

$$
I_{C_{\epsilon}}+I_{A B}^{\prime}+I_{C_{\varepsilon}}+I_{C D}^{\prime}+I_{C_{1 \varepsilon_{1}}^{\prime}}^{\prime}+I_{E F}^{\prime}=0
$$

From here we get that

$$
I_{C_{\varepsilon}}=-I_{A B}^{\prime}-I_{C_{\varepsilon}}-I_{C D}^{\prime}-I_{C_{1 \varepsilon_{1}}^{\prime}}^{\prime}-I_{E F}^{\prime} .
$$

Thus, it is possible to substitute integration on the arc of the circle $C_{\epsilon}$ by integration on the remaining parts of the contour $\Gamma^{\prime}$.

We consider each integral in the right part of this expression. The integral $I_{C_{\varepsilon}}$ has already been considered by us earlier in this lemma. Using (42) we obtain that in the case considered

$$
\lim _{\varepsilon \rightarrow 0} I_{C_{\varepsilon}}=0, \quad \mu_{R}<1+1 / \rho .
$$

Consider the integral $I_{A B}^{\prime}$. Representing the complex number $\zeta$ in the form $\zeta=r e^{i \varphi}$ and using (51) we obtain

$$
I_{A B}^{\prime}=\int_{A B} \frac{\phi_{\rho, \mu}(\zeta, z)}{\zeta-1} d \zeta=\left.\int_{1+\epsilon}^{\varepsilon} \frac{\phi_{\rho, \mu}\left(r e^{i \varphi}, z\right)}{r e^{i \varphi}-1} e^{i \varphi} d r\right|_{\varphi=\delta_{2 \rho}-\pi}=\int_{1+\epsilon}^{\varepsilon} \frac{\phi_{\rho, \mu}\left(r e^{i\left(\delta_{2 \rho}-\pi\right)}, z\right)}{r e^{i\left(\delta_{2 \rho}-\pi\right)}-1} e^{i\left(\delta_{2 \rho}-\pi\right)} d r .
$$

We introduce the notation

$$
\frac{\phi_{\rho, \mu}\left(r e^{i(\delta-\pi)}, z\right)}{r e^{i(\delta-\pi)}-1} e^{i(\delta-\pi)}=K_{\rho, \mu}^{\prime}(r, \delta, z)
$$

Now we transform the function $K_{\rho, \mu}^{\prime}(r, \delta, z)$. Using the definition for $\phi_{\rho, \mu}(\zeta, z)$ (see (13)) and getting rid of the complexity in the denominator we obtain

$$
\begin{aligned}
& K_{\rho, \mu}^{\prime}(r, \delta, z)=\frac{\rho}{2 \pi i} \frac{\exp \left\{\left(z r e^{i(\delta-\pi)}\right)^{\rho}\right\}\left(z r e^{i(\delta-\pi)}\right)^{\rho(1-\mu)} e^{i(\delta-\pi)}\left(r e^{-i(\delta-\pi)}-1\right)}{\left(r e^{i(\delta-\pi)}-1\right)\left(r e^{-i(\delta-\pi)}-1\right)}= \\
& \frac{\rho}{2 \pi i} \frac{\exp \left\{(z r)^{\rho} e^{-i \rho \pi}[\cos (\rho \delta)+i \sin (\rho \delta)]\right\}\left(z r e^{-i \pi}\right)^{\rho(1-\mu)} e^{i \rho(1-\mu) \delta}\left(r-e^{i(\delta-\pi)}\right)}{r^{2}-2 r \cos (\delta-\pi)+1}= \\
& \frac{\rho}{2 \pi i} \frac{\exp \left\{(z r)^{\rho} e^{-i \rho \pi} \cos (\rho \delta)\right\}\left(z r e^{-i \pi}\right)^{\rho(1-\mu)}}{r^{2}+2 r \cos \delta+1} \exp \left\{i\left[(z r)^{\rho} e^{-i \pi \rho} \sin (\rho \delta)+\rho(1-\mu) \delta\right]\right\}\left(r+e^{i \delta}\right)
\end{aligned}
$$

By introducing the notation $\eta(r, \varphi, z)=(z r)^{\rho} e^{-i \pi \rho} \sin (\rho \delta)+\rho(1-\mu) \delta$, that coincides with (10) we obtain that $K_{\rho, \mu}^{\prime}(r, \delta, z)$ can be represented in the form

$$
K_{\rho, \mu}^{\prime}(r, \delta, z)=\frac{\rho}{2 \pi i} \frac{\exp \left\{(z r)^{\rho} e^{-i \rho \pi} \cos (\rho \delta)\right\}\left(z r e^{-i \pi}\right)^{\rho(1-\mu)} e^{i \eta(r, \varphi, z)}\left(r+e^{i \delta}\right)}{r^{2}+2 r \cos \delta+1}
$$


Thus, for the integral $I_{A B}^{\prime}$ we get

$$
I_{A B}^{\prime}=\int_{1+\epsilon}^{\varepsilon} K_{\rho, \mu}^{\prime}\left(r, \delta_{2 \rho}, z\right) d r
$$

Now consider the integral $I_{C D}^{\prime}$. Representing the complex number $\zeta$ in the form $\zeta=r e^{i \varphi}$ and using (51) we get

$$
I_{C D}^{\prime}=\int_{C D} \frac{\phi_{\rho, \mu}(\zeta, z)}{\zeta-1} d \zeta=\left.\int_{\varepsilon}^{1-\varepsilon_{1}} \frac{\phi_{\rho, \mu}\left(r e^{i \varphi}, z\right)}{r e^{i \varphi}-1} e^{i \varphi} d r\right|_{\varphi=-\pi-\pi}=\int_{\varepsilon}^{1-\varepsilon_{1}} \frac{\phi_{\rho, \mu}\left(r e^{i(-\pi-\pi)}, z\right)}{r e^{i(-\pi-\pi)}-1} e^{i(-\pi-\pi)} d r .
$$

Comparing this expression with (55), we notice that the integrand of the integral obtained is similar to the integrand of the integral (55). Therefore, using (56) we get that the integrand can be represented in the form (57). Thus, for the integral $I_{C D}^{\prime}$ we obtain

$$
I_{C D}^{\prime}=\int_{\varepsilon}^{1-\varepsilon_{1}} K_{\rho, \mu}^{\prime}(r,-\pi, z) d r
$$

Similarly, using (51) for the integral $I_{E F}^{\prime}$ we obtain

$$
I_{E F}^{\prime}=\int_{E F} \frac{\phi_{\rho, \mu}(\zeta, z)}{\zeta-1} d \zeta=\left.\int_{1+\varepsilon_{1}}^{1+\epsilon} \frac{\phi_{\rho, \mu}\left(r e^{i \varphi}, z\right)}{r e^{i \varphi}-1} e^{i \varphi} d r\right|_{\varphi=-\pi-\pi}=\int_{1+\varepsilon_{1}}^{1+\epsilon} \frac{\phi_{\rho, \mu}\left(r e^{i(-\pi-\pi)}, z\right)}{r e^{i(-\pi-\pi)}-1} e^{i(-\pi-\pi)} d r .
$$

Using now (56) and (57) the integral $I_{E F}^{\prime}$ takes the form

$$
I_{E F}^{\prime}=\int_{1+\varepsilon_{1}}^{1+\epsilon} K_{\rho, \mu}^{\prime}(r,-\pi, z) d r .
$$

In the sum (52) it remains to consider the integral $I_{C_{1 \varepsilon_{1}}^{\prime}}^{\prime}$. This integral is taken along the arc of the circle $C_{1 \varepsilon_{1}}^{\prime}$ with the center in the point $\zeta=1$ (at that $\arg \zeta=-2 \pi$ ) and radius $\varepsilon_{1}$. The contour is traversed in the direction from the point $D$ to the point $E$ (see Figure 3). As it was shown earlier, the arc of the circle $C_{1 \varepsilon_{1}}^{\prime}$ in the plane $\zeta$ can be given in the form (see (51))

$$
C_{1 \varepsilon_{1}}^{\prime}=\left\{\zeta: \begin{array}{c}
\arg \zeta=\arctan \left(\frac{\tau \sin \psi}{\tau \cos \psi+1}\right)+k \pi,|\zeta|=\sqrt{\tau^{2}+2 \tau \cos \psi+1} \\
-\pi \geqslant \psi \geqslant-2 \pi, \tau=\varepsilon_{1}, k=-2
\end{array}\right\}
$$

As one can see, the arc traverse in the direction from the point $D$ to the point $E$ corresponds to a change of the parameter $\psi$ from $-\pi$ to $-2 \pi$. Thus, in the case under consideration the contour $C_{1 \varepsilon_{1}}^{\prime}$ on the complex plane $\zeta$ can be represented in the form

$$
\zeta=r\left(\varepsilon_{1}, \psi\right) e^{i \varphi\left(\varepsilon_{1}, \psi,-2\right)},
$$

where $-\pi \geqslant \psi \geqslant-2 \pi, 0<\varepsilon_{1}<1$ and

$$
\begin{aligned}
& r(\tau, \psi)=\sqrt{\tau^{2}+2 \tau \cos \psi+1} \\
& \varphi(\tau, \psi, k)=\arctan \left(\frac{\tau \sin \psi}{\tau \cos \psi+1}\right)+k \pi
\end{aligned}
$$


Representing now in the integral $I_{C_{1 \varepsilon_{1}}^{\prime}}^{\prime}$ the complex number $\zeta$ in the form (62) we find

$$
I_{C_{1 \varepsilon_{1}}^{\prime}}^{\prime}=\int_{C_{1 \varepsilon_{1}}^{\prime}} \frac{\phi_{\rho, \mu}(\zeta, z)}{\zeta-1} d \zeta=-i \varepsilon_{1} \int_{-2 \pi}^{-\pi} \frac{\phi_{\rho, \mu}\left(r\left(\varepsilon_{1}, \psi\right) e^{i \varphi\left(\varepsilon_{1}, \psi,-2\right)}, z\right)}{r\left(\varepsilon_{1}, \psi\right) e^{i \varphi\left(\varepsilon_{1}, \psi,-2\right)}-1} e^{i \psi} d \psi .
$$

One should pay attention that traversing along the arc of the circle $C_{1 \varepsilon_{1}}^{\prime}$ in the direction from the point $D$ to the point $E$ (see Figure 3) corresponds to the negative traversing direction along the contour. Here the value of the parameter $\psi=-\pi$ corresponds to the point $D$ and the value of the parameter $\psi=-2 \pi$ to the point $E$. That is why in the integral derived one must transpose the limits of integration.

We now consider the integrand of this integral. We introduce the following notation for it

$$
i \tau \frac{\phi_{\rho, \mu}\left(r(\tau, \psi) e^{i \varphi(\tau, \psi, k)}, z\right)}{r(\tau, \psi) e^{i \varphi(\tau, \psi, k)}-1} e^{i \psi}=P_{\rho, \mu}^{\prime}(\tau, \psi, k, z) .
$$

We transform this expression using (13) and getting rid of the complexity in the denominator we get

$$
\begin{gathered}
P_{\rho, \mu}^{\prime}(\tau, \psi, k, z)=\frac{\rho \tau}{2 \pi} \frac{\exp \left\{\left(z r(\tau, \psi) e^{i \varphi(\tau, \psi, k)}\right)^{\rho}\right\}\left(z r(\tau, \psi) e^{i \varphi(\tau, \psi, k)}\right)^{\rho(1-\mu)} e^{i \psi}}{r(\tau, \psi) e^{i \varphi(\tau, \psi, k)}-1} \\
=\frac{\rho \tau}{2 \pi} \frac{\exp \left\{(z r(\tau, \psi))^{\rho}(\cos (\rho \varphi(\tau, \psi, k))+i \sin (\rho \varphi(\tau, \psi, k)))\right\}}{\left(r(\tau, \psi) e^{i \varphi(\tau, \psi, k)}-1\right)\left(r(\tau, \psi) e^{-i \varphi(\tau, \psi, k)}-1\right)} \times \\
\times(z r(\tau, \psi))^{\rho(1-\mu)} e^{i \rho(1-\mu) \varphi(\tau, \psi, k)}\left(r(\tau, \psi) e^{-i \varphi(\tau, \psi, k)}-1\right) e^{i \psi} \\
=\frac{\rho \tau}{2 \pi} \frac{\exp \left\{(z r(\tau, \psi))^{\rho} \cos (\rho \varphi(\tau, \psi, k))\right\}(z r(\tau, \varphi))^{\rho(1-\mu)}}{(r(\tau, \psi))^{2}-2 r(\tau, \psi) \cos \varphi(\tau, \psi, k)+1} \times \\
\times \exp \left\{i\left[(z r(\tau, \psi))^{\rho} \sin (\rho \varphi(\tau, \psi, k))+\rho(1-\mu) \varphi(\tau, \psi, k)\right]\right\}\left(r(\tau, \psi) e^{-i \varphi(\tau, \psi, k)}-1\right) e^{i \psi} \\
=\frac{\rho \tau}{2 \pi} \frac{\exp \left\{(z r(\tau, \psi))^{\rho} \cos (\rho \varphi(\tau, \psi, k))\right\}(z r(\tau, \varphi))^{\rho(1-\mu)}}{(r(\tau, \psi))^{2}-2 r(\tau, \psi) \cos \varphi(\tau, \psi, k)+1} e^{i\left[\chi^{\prime}(\tau, \psi, k, z)+\psi\right]}\left(r(\tau, \psi) e^{-i \varphi(\tau, \psi, k)}-1\right),
\end{gathered}
$$

where

$$
\chi^{\prime}(\tau, \psi, k, z)=(z r(\tau, \psi))^{\rho} \sin (\rho \varphi(\tau, \psi, k))+\rho(1-\mu) \varphi(\tau, \psi, k) .
$$

As a result the integral (64) takes the form

$$
I_{C_{1 \varepsilon_{1}}^{\prime}}^{\prime}=-\int_{-2 \pi}^{-\pi} P_{\rho, \mu}^{\prime}\left(\varepsilon_{1}, \psi,-2, z\right) d \psi
$$

Now we get back to the expression (53) and let $\varepsilon \rightarrow 0$ in this expression. It is necessary to point out that the integrals $I_{C_{\epsilon}}, I_{C_{1 \varepsilon_{1}}^{\prime}}^{\prime}$ and $I_{E F}^{\prime}$ do not depend on $\varepsilon$ and, consequently, they will not change with such a passage to the limit. In view of this, we have

$$
I_{C_{\varepsilon}}=-\lim _{\varepsilon \rightarrow 0} I_{A B}^{\prime}-\lim _{\varepsilon \rightarrow 0} I_{C_{\varepsilon}}-\lim _{\varepsilon \rightarrow 0} I_{C D}^{\prime}-I_{C_{1 \varepsilon_{1}}^{\prime}}^{\prime}-I_{E F}^{\prime} .
$$


Taking into account (54) and using the expressions (58), (59), (60) and (67) we get

$$
\begin{aligned}
I_{C_{\epsilon}}=-\lim _{\varepsilon \rightarrow 0} \int_{1+\epsilon}^{\varepsilon} K_{\rho, \mu}^{\prime}\left(r, \delta_{2 \rho}, z\right) d r & -\lim _{\varepsilon \rightarrow 0} \int_{\varepsilon}^{1-\varepsilon_{1}} K_{\rho, \mu}^{\prime}(r,-\pi, z) d r \\
& +\int_{-2 \pi}^{-\pi} P_{\rho, \mu}^{\prime}\left(\varepsilon_{1}, \psi,-2, z\right) d \psi-\int_{1+\varepsilon_{1}}^{1+\epsilon} K_{\rho, \mu}^{\prime}(r,-\pi, z) d r, \quad \Re \mu<1+1 / \rho .
\end{aligned}
$$

From this we obtain

$$
\begin{aligned}
I_{C_{\epsilon}}=-\int_{1+\epsilon}^{0} K_{\rho, \mu}^{\prime}\left(r, \delta_{2 \rho}, z\right) d r & -\int_{0}^{1-\varepsilon_{1}} K_{\rho, \mu}^{\prime}(r,-\pi, z) d r \\
& +\int_{-2 \pi}^{-\pi} P_{\rho, \mu}^{\prime}\left(\varepsilon_{1}, \psi,-2, z\right) d \psi-\int_{1+\varepsilon_{1}}^{1+\epsilon} K_{\rho, \mu}^{\prime}(r,-\pi, z) d r, \quad \Re \mu<1+1 / \rho .
\end{aligned}
$$

Now we get back to the Mittag-Leffler function. Assuming that $\delta_{1 \rho}=\pi$ the contour of integration $\gamma_{\zeta}$ in (37) takes the form

$$
\gamma_{\zeta}=\left\{\begin{array}{l}
S_{1}=\{\zeta: \arg \zeta=-2 \pi,|\zeta| \geqslant 1+\epsilon\} \\
C_{\epsilon}=\left\{\zeta:-2 \pi \leqslant \arg \zeta \leqslant \delta_{2 \rho}-\pi,|\zeta|=1+\epsilon\right\} \\
S_{2}=\left\{\zeta: \arg \zeta=\delta_{2 \rho}-\pi,|\zeta| \geqslant 1+\epsilon\right\}
\end{array}\right.
$$

By calculating (37) directly and representing a complex number $\zeta$ in the form $\zeta=r e^{i \varphi}$ we obtain

$$
\begin{aligned}
& E_{\rho, \mu}(z)=\int_{S_{1}} \frac{\phi_{\rho, \mu}(\zeta, z)}{\zeta-1} d \zeta+\int_{C_{\epsilon}} \frac{\phi_{\rho, \mu}(\zeta, z)}{\zeta-1} d \zeta+\int_{S_{1}} \frac{\phi_{\rho, \mu}(\zeta, z)}{\zeta-1} d \zeta \\
&=\left.\int_{\infty}^{1+\epsilon} \frac{\phi_{\rho, \mu}\left(r e^{i \varphi}, z\right)}{r e^{i \varphi}-1} e^{i \varphi} d r\right|_{\varphi=-\pi-\pi}+I_{C_{\epsilon}}+\left.\int_{1+\epsilon}^{\infty} \frac{\phi_{\rho, \mu}\left(r e^{i \varphi}, z\right)}{r e^{i \varphi}-1} e^{i \varphi} d r\right|_{\varphi=\delta_{2 \rho}-\pi} \\
& \quad=\int_{\infty}^{1+\epsilon} \frac{\phi_{\rho, \mu}\left(r e^{i(-\pi-\pi)}, z\right)}{r e^{i(-\pi-\pi)}-1} e^{i(-\pi-\pi)} d r+I_{C_{\epsilon}}+\int_{1+\epsilon}^{\infty} \frac{\phi_{\rho, \mu}\left(r e^{i\left(\delta_{2 \rho}-\pi\right)}, z\right)}{r e^{i\left(\delta_{2 \rho}-\pi\right)}-1} e^{i\left(\delta_{2 \rho}-\pi\right)} d r
\end{aligned}
$$

using the notation (56), this expression can be written in the form

$$
E_{\rho, \mu}(z)=\int_{\infty}^{1+\epsilon} K_{\rho, \mu}^{\prime}(r,-\pi, z) d r+I_{C_{\epsilon}}+\int_{1+\epsilon}^{\infty} K_{\rho, \mu}^{\prime}\left(r, \delta_{2 \rho}, z\right) d r,
$$

where the form of the functions $K_{\rho, \mu}^{\prime}(r, \delta, z)$ is defined by (57). Now we will make use of the representation here (68) for the integral $I_{C_{\epsilon}}$. As a result, we obtain

$$
\begin{aligned}
& E_{\rho, \mu}(z)=\int_{\infty}^{1+\epsilon} K_{\rho, \mu}^{\prime}(r,-\pi, z) d r-\int_{1+\epsilon}^{0} K_{\rho, \mu}^{\prime}\left(r, \delta_{2 \rho}, z\right) d r-\int_{0}^{1-\varepsilon_{1}} K_{\rho, \mu}^{\prime}(r,-\pi, z) d r \\
& \quad+\int_{-2 \pi}^{-\pi} P_{\rho, \mu}^{\prime}\left(\varepsilon_{1}, \psi,-2, z\right) d \psi-\int_{1+\varepsilon_{1}}^{1+\epsilon} K_{\rho, \mu}^{\prime}(r,-\pi, z) d r+\int_{1+\epsilon}^{\infty} K_{\rho, \mu}^{\prime}\left(r, \delta_{2 \rho}, z\right) d r \\
& =\int_{0}^{\infty} K_{\rho, \mu}^{\prime}\left(r, \delta_{2 \rho}, z\right) d r-\int_{0}^{1-\varepsilon_{1}} K_{\rho, \mu}^{\prime}(r,-\pi, z) d r+\int_{-2 \pi}^{-\pi} P_{\rho, \mu}^{\prime}\left(\varepsilon_{1}, \psi,-2, z\right) d \psi-\int_{1+\varepsilon_{1}}^{\infty} K_{\rho, \mu}^{\prime}(r,-\pi, z) d r,
\end{aligned}
$$

where $\Re \mu<1+1 / \rho$. It remains to consider how to change the condition (2) in this case. Since, in the case considered $\frac{1}{2}<\rho \leqslant 1, \delta_{1 \rho}=\pi, \pi /(2 \rho)<\delta_{2 \rho} \leqslant \pi$, then the condition (2) takes the form $\frac{\pi}{2 \rho}-\delta_{2 \rho}+\pi<$ $\arg z<-\frac{\pi}{2 \rho}+2 \pi$. Thus, we have obtained the statement of the theorem for the second case. 
Case 3. Consider now the case $1 / 2<\rho \leqslant 1, \pi /(2 \rho)<\delta_{1 \rho} \leqslant \pi, \delta_{2 \rho}=\pi$. In this case the segment $\Gamma_{2}$ of the auxiliary contour $\Gamma$ (see Figure 2) will run along the positive part of a real axis and, thus, will run through a singular point $\zeta^{\prime \prime}=1$. It should be pointed out that in this case an argument of points of the segment is equal to $\arg \zeta=0$. Consequently, in this case $\arg \zeta^{\prime \prime}=0$. That is why, as in the previous case, we will change the contour $\Gamma$ in such a way that the contour bypasses this singular point leaving it outside the contour. The contour formed $\Gamma^{\prime \prime}$ (Figure 4) consists of the arc of the circle $C_{\epsilon}=\{\zeta$ : $\left.-\delta_{1 \rho}-\pi \leqslant \arg \zeta \leqslant 0,|\zeta|=1+\epsilon\right\}$ with the center in the point $\zeta=0$ and radius $1+\epsilon, \epsilon>0$, the segment $A B=\left\{\zeta: \arg \zeta=0,1+\epsilon \geqslant|\zeta| \geqslant 1+\varepsilon_{1}\right\}$, the arc of the circle $C_{1 \varepsilon_{1}}^{\prime \prime}$ with the center in the point $\zeta^{\prime \prime}=1$, $\left(\arg \zeta^{\prime \prime}=0\right)$ and radius $\varepsilon_{1},\left(0<\varepsilon_{1}<1, \varepsilon_{1}<\epsilon\right)$, the segment $C D=\left\{\zeta: \arg \zeta=0,1-\varepsilon_{1} \geqslant|\zeta| \geqslant \varepsilon\right\}$, the arc of the circle $C_{\varepsilon}=\left\{\zeta: 0 \geqslant \arg \zeta \geqslant-\delta_{1 \rho}-\pi,|\zeta|=\varepsilon\right\}$ with the center at the origin of coordinates and radius $\varepsilon>0$ and $\varepsilon<1-\varepsilon_{1}$ and the segment $E F=\left\{\zeta: \arg \zeta=-\delta_{1 \rho}-\pi, \varepsilon \leqslant|\zeta| \leqslant 1+\epsilon\right\}$.

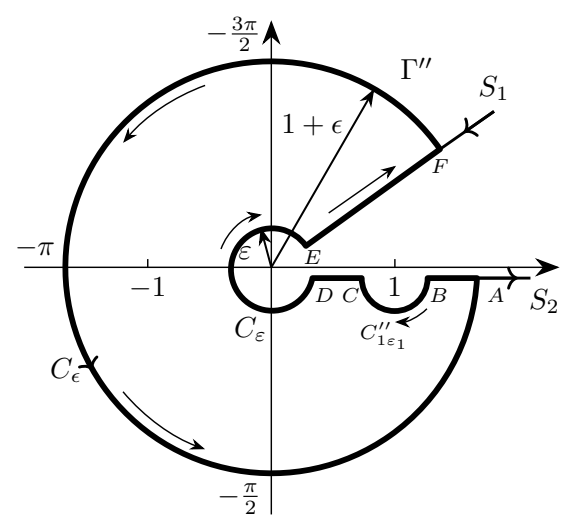

Figure 4. The auxiliary contour of integration $\Gamma^{\prime \prime}$.

As one can see, only the arc of the circle $C_{1 \varepsilon_{1}}^{\prime \prime}$ remains non-parametric. For its parametrization we will fulfill the procedure as in the previous case. Considering the conformal mapping $u=\zeta-1$ and representing the complex number $u$ in the form $u=\tau e^{i \psi}$ we get that the arc of the circle $C_{1 \varepsilon_{1}}^{\prime \prime}$ can be represented in the form (50), where the parameter $\psi$ varies within the limits from $-\pi$ to 0 . Note that the value of the parameter $\psi=-\pi$ corresponds to the point $C$ and the value $\psi=0$ to the point $B$. It should be pointed out that the center of a circle lies in the point $\zeta^{\prime \prime}=1$ with $\arg \zeta^{\prime \prime}=0$. Consequently, in the formulas (50) one should choose the principal branch $\arctan (x)$, i.e., to take $k=0$. As a result, an arc of a circle $C_{1 \varepsilon_{1}}^{\prime \prime}$ can be represented in the form

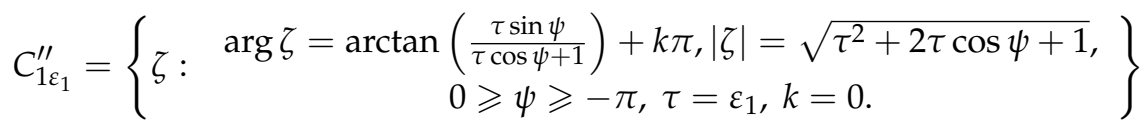

In view of the foregoing, the contour $\Gamma^{\prime \prime}$ is written in the form

$$
\Gamma^{\prime \prime}=\left\{\begin{array}{c}
C_{\epsilon}=\left\{\zeta:-\delta_{1 \rho}-\pi \leqslant \arg \zeta \leqslant 0,|\zeta|=1+\epsilon\right\} \\
A B=\left\{\zeta: \arg \zeta=0,1+\epsilon \geqslant|\zeta| \geqslant 1+\varepsilon_{1}\right\} \\
C_{1 \varepsilon_{1}}^{\prime \prime}=\left\{\begin{array}{c}
\arg \zeta=\arctan \left(\frac{\tau \sin \psi}{\tau \cos \psi-1}+k \pi\right) \\
\zeta: \quad|\zeta|=\sqrt{\tau^{2}+2 \tau \cos \psi+1} \\
0 \geqslant \arg \zeta \geqslant-\pi, \tau=\varepsilon_{1}, k=0
\end{array}\right\} \\
C D=\left\{\zeta: \arg \zeta=0,1-\varepsilon_{1} \geqslant|\zeta| \geqslant \varepsilon\right\} \\
C_{\varepsilon}=\left\{\zeta: 0 \geqslant \arg \zeta \geqslant-\delta_{1 \rho}-\pi,|\zeta|=\varepsilon\right\} \\
E F=\left\{\zeta: \arg \zeta=-\delta_{1 \rho}-\pi, \varepsilon \leqslant|\zeta| \leqslant 1+\epsilon\right\} .
\end{array}\right.
$$


The contour is traversed in a positive direction.

We consider now an auxiliary integral

$$
I^{\prime \prime}=\int_{\Gamma^{\prime \prime}} \frac{\phi_{\rho, \mu}(\zeta, z)}{\zeta-1} d \zeta
$$

In a similar way to how it was done in the previous case, we calculate this integral. Using the definition of the contour $\Gamma^{\prime \prime}$ we have

$$
\begin{aligned}
I^{\prime \prime}=\int_{C_{e}} \frac{\phi_{\rho, \mu}(\zeta, z)}{\zeta-1} d \zeta+ & \int_{A B} \frac{\phi_{\rho, \mu}(\zeta, z)}{\zeta-1} d \zeta+\int_{C_{1 \varepsilon_{1}}^{\prime \prime}} \frac{\phi_{1, \mu}(\zeta, z)}{\zeta-1} d \zeta+\int_{C D} \frac{\phi_{\rho, \mu}(\zeta, z)}{\zeta-1} d \zeta \\
& \quad+\int_{C_{\varepsilon}} \frac{\phi_{\rho, \mu}(\zeta, z)}{\zeta-1} d \zeta+\int_{E F} \frac{\phi_{\rho, \mu}(\zeta, z)}{\zeta-1} d \zeta=I_{C_{\varepsilon}}+I_{A B}^{\prime \prime}+I_{C_{1 \varepsilon_{1}}^{\prime \prime}}^{\prime \prime}+I_{C D}^{\prime \prime}+I_{C_{\varepsilon}}+I_{E F}^{\prime \prime} .
\end{aligned}
$$

It is clear from this expression that inside the region limited by the contour $\Gamma^{\prime \prime}$ the integrand of the integral $I^{\prime \prime}$ is an analytical and continuous function of a variable $\zeta$. Consequently, according to the Cauchy theorem

$$
I_{C_{\epsilon}}+I_{A B}^{\prime \prime}+I_{C_{1 \varepsilon_{1}}^{\prime \prime}}^{\prime \prime}+I_{C D}^{\prime \prime}+I_{C_{\varepsilon}}+I_{E F}^{\prime \prime}=0 .
$$

From this we obtain that

$$
I_{C_{\varepsilon}}=-I_{A B}^{\prime \prime}-I_{C_{1 \varepsilon_{1}}^{\prime \prime}}^{\prime \prime}-I_{C D}^{\prime \prime}-I_{C_{\varepsilon}}-I_{E F}^{\prime \prime}
$$

We consider now each of the integrals in the right part separately.

The integral $I_{C_{\varepsilon}}$ was considered by us earlier. Using (42), we obtain

$$
\lim _{\varepsilon \rightarrow 0} I_{C_{\varepsilon}}=0, \quad \Re \mu<1+1 / \rho .
$$

Now consider the integral $I_{A B}^{\prime \prime}$. Representing $\zeta$ in the form $\zeta=r e^{i \varphi}$ we find

$$
I_{A B}^{\prime \prime}=\left.\int_{1+\epsilon}^{1+\varepsilon_{1}} \frac{\phi_{\rho, \mu}\left(r e^{i \varphi}, z\right)}{r e^{i \varphi}-1} e^{i \varphi} d r\right|_{\pi-\pi}=\int_{1+\epsilon}^{1+\varepsilon_{1}} \frac{\phi_{\rho, \mu}\left(r e^{i(\pi-\pi)}, z\right)}{r e^{i(\pi-\pi)}-1} e^{i(\pi-\pi)} d r .
$$

From this it is clear that this integral is similar to the integral (55). That is why one can use the study results of this integral. Using (57), we obtain

$$
I_{A B}^{\prime \prime}=\int_{1+\epsilon}^{1+\varepsilon_{1}} K_{\rho, \mu}^{\prime}(r, \pi, z) d r .
$$

In the same way, using (70), (56) and (57) for the integrals $I_{C D}^{\prime \prime}$ and $I_{E F}^{\prime \prime}$ we find

$$
I_{C D}^{\prime \prime}=\int_{1-\varepsilon_{1}}^{\varepsilon} K_{\rho, \mu}^{\prime}(r, \pi, z) d r, \quad I_{E F}^{\prime \prime}=\int_{\varepsilon}^{1+\epsilon} K_{\rho, \mu}^{\prime}(r, \pi, z) d r
$$

It remains to consider the integral $I_{C_{1 \varepsilon_{1}}^{\prime \prime}}$. This integral is taken along the arc of the $C_{1 \varepsilon_{1}}^{\prime \prime}$ with the center in the point $\zeta^{\prime \prime}=1$, where $\arg \zeta^{\prime \prime}=0$. This means that the contour is traversed from the point $B$ of the complex plane $\zeta$ in which $\arg \zeta_{B}=0$ to the point $C$ in which $\arg \zeta_{C}=0$. In other words, the starting and ending points of this contour have the same value of the argument. As it was shown above, the arc of a circle $C_{1 \varepsilon_{1}}^{\prime \prime}$ can be represented in the form (69). Traversing this arc in the direction from the point $B$ to the 
point $C$ corresponds to a change of the parameter $\psi$ from 0 to $-\pi$. Thus, the points of the arc of the circle $C_{1 \varepsilon_{1}}^{\prime \prime}$ can be represented in the form

$$
\zeta=r\left(\varepsilon_{1}, \psi\right) e^{i \varphi\left(\varepsilon_{1}, \psi, 0\right)},
$$

where $r(\tau, \psi)$ and $\varphi(\tau, \psi, k)$ have the form (63).

Using now the representation (75), in the integral $I_{C_{1 \varepsilon_{1}}^{\prime \prime}}$ we obtain

$$
I_{C_{1 \varepsilon_{1}}^{\prime \prime}}^{\prime \prime}=\left.\int_{0}^{-\pi} \frac{\phi_{\rho, \mu}\left(r(\tau, \psi) e^{i \varphi(\tau, \psi, 0)}, z\right) i \tau e^{i \psi}}{r(\tau, \psi) e^{i \varphi(\tau, \psi, 0)}-1} d \psi\right|_{\tau=\varepsilon_{1}}=-i \varepsilon_{1} \int_{-\pi}^{0} \frac{\phi_{\rho, \mu}\left(r\left(\varepsilon_{1}, \psi\right) e^{i \varphi\left(\varepsilon_{1}, \psi, 0\right)}, z\right) e^{i \psi}}{r(\tau, \psi) e^{i \varphi(\tau, \psi, 0)}-1} d \psi .
$$

Comparing this expression with (64) it is clear that these two integrals are similar. Therefore, using (65) and (66) for the integral $I_{C_{1 \varepsilon_{1}}^{\prime \prime}}^{\prime \prime}$ we get

$$
I_{C_{1 \varepsilon_{1}}^{\prime \prime}}^{\prime \prime}=-\int_{-\pi}^{0} P_{\rho, \mu}^{\prime}\left(\varepsilon_{1}, \psi, 0, z\right) d \psi
$$

We return to the expression (71) and let $\varepsilon \rightarrow 0$ in this expression. Note that the integrals $I_{C_{\varepsilon}}, I_{A B}^{\prime \prime} I_{C_{1 \varepsilon_{1}}^{\prime \prime}}^{\prime \prime}$ do not depend on $\varepsilon$ and, consequently, with such a passage to the limit they will not change. As a result we obtain

$$
I_{C_{\varepsilon}}=-I_{A B}^{\prime \prime}-I_{C_{1 \varepsilon_{1}}^{\prime \prime}}^{\prime \prime}-\lim _{\varepsilon \rightarrow 0} I_{C D}^{\prime \prime}-\lim _{\varepsilon \rightarrow 0} I_{C_{\varepsilon}}-\lim _{\varepsilon \rightarrow 0} I_{E F}^{\prime \prime} .
$$

Using here the expressions (72)-(74), (76) we obtain

$$
\begin{aligned}
I_{C_{\epsilon}}=-\int_{1+\epsilon}^{1+\varepsilon_{1}} K_{\rho, \mu}^{\prime}(r, \pi, z) d r+ & \int_{-\pi}^{0} P_{\rho, \mu}^{\prime}\left(\varepsilon_{1}, \psi, 0, z\right) d \psi \\
& -\lim _{\varepsilon \rightarrow 0} \int_{1-\varepsilon_{1}}^{\varepsilon} K_{\rho, \mu}^{\prime}(r, \pi, z) d r-\lim _{\varepsilon \rightarrow 0} \int_{\varepsilon}^{1+\epsilon} K_{\rho, \mu}^{\prime}(r, \pi, z) d r, \quad \Re \mu<1+1 / \rho .
\end{aligned}
$$

As a result, performing the passage to the limit we have

$$
\begin{aligned}
I_{C_{\epsilon}}=-\int_{1+\epsilon}^{1+\varepsilon_{1}} K_{\rho, \mu}^{\prime}(r, \pi, z) d r+\int_{-\pi}^{0} P_{\rho, \mu}^{\prime}\left(\varepsilon_{1}, \psi, 0, z\right) d \psi- & \int_{1-\varepsilon_{1}}^{0} K_{\rho, \mu}^{\prime}(r, \pi, z) d r \\
& -\int_{0}^{1+\epsilon} K_{\rho, \mu}^{\prime}(r, \pi, z) d r, \quad \Re \mu<1+1 / \rho .
\end{aligned}
$$

We return now to the Mittag-Leffler function. Assuming that $\delta_{2 \rho}=\pi$ the contour of integration $\gamma_{\zeta}$ in (37) takes the form

$$
\gamma_{\zeta}=\left\{\begin{array}{l}
S_{1}=\left\{\zeta: \arg \zeta=-\delta_{1 \rho}-\pi,|\zeta| \geqslant 1+\epsilon\right\} \\
C_{\epsilon}=\left\{\zeta:-\delta_{1 \rho}-\pi \leqslant \arg \zeta \leqslant 0,|\zeta|=1+\epsilon\right\} \\
S_{2}=\{\zeta: \arg \zeta=0,|\zeta| \geqslant 1+\epsilon\}
\end{array}\right.
$$

and the condition (2) will be written in the form $\frac{\pi}{2 \rho}<\arg z<-\frac{\pi}{2 \rho}+\delta_{1 \rho}+\pi$.

By direct calculating (37) and representing the complex number $\zeta$ in the form $\zeta=r e^{i \varphi}$ we get 


$$
\begin{aligned}
E_{\rho, \mu}(z)=\int_{S_{1}} \frac{\phi_{\rho, \mu}(\zeta, z)}{\zeta-1} d \zeta+\int_{C_{\epsilon}} \frac{\phi_{\rho, \mu}(\zeta, z)}{\zeta-1} d \zeta+\int_{S_{1}} \frac{\phi_{\rho, \mu}(\zeta, z)}{\zeta-1} d \zeta & \quad+I_{C_{\epsilon}}+\left.\left.\int_{1+\epsilon}^{\infty} \frac{\phi_{\rho, \mu}\left(r e^{i \varphi}, z\right)}{r e^{i \varphi}-1} e^{i \varphi} d r\right|_{\varphi=\pi-\pi} \int_{\infty}^{1+\epsilon} \frac{\phi_{\rho, \mu}\left(r e^{i \varphi}, z\right)}{r e^{i \varphi}-1} e^{i \varphi} d r\right|_{\varphi=-\delta_{1 \rho}-\pi} \\
& =\int_{\infty}^{1+\epsilon} \frac{\phi_{\rho, \mu}\left(r e^{i\left(-\delta_{1 \rho}-\pi\right)}, z\right)}{r e^{i\left(-\delta_{1 \rho}-\pi\right)}-1} e^{i\left(-\delta_{1 \rho}-\pi\right)} d r+I_{C_{\epsilon}}+\int_{1+\epsilon}^{\infty} \frac{\phi_{\rho, \mu}\left(r e^{i(\pi-\pi)}, z\right)}{r e^{i(\pi-\pi)}-1} e^{i(\pi-\pi)} d r
\end{aligned}
$$

using the notation (56) this expression can be written in the form

$$
E_{\rho, \mu}(z)=\int_{\infty}^{1+\epsilon} K_{\rho, \mu}^{\prime}\left(r,-\delta_{1 \rho}, z\right) d r+I_{C_{\epsilon}}+\int_{1+\epsilon}^{\infty} K_{\rho, \mu}^{\prime}(r, \pi, z) d r
$$

where the form of functions $K_{\rho, \mu}^{\prime}(r, \delta, z)$ is defined as (57). We substitute the integral $I_{C_{e}}$ in this expression by the expression (77). As a result, we obtain

$$
\begin{aligned}
E_{\rho, \mu}(z)=\int_{0}^{1-\varepsilon_{1}} K_{\rho, \mu}^{\prime}(r, \pi, z) d r+\int_{-\pi}^{0} P_{\rho, \mu}^{\prime}\left(\varepsilon_{1}, \psi, 0, z\right) d \psi & \\
& +\int_{1+\varepsilon_{1}}^{\infty} K_{\rho, \mu}^{\prime}(r, \pi, z) d r-\int_{0}^{\infty} K_{\rho, \mu}^{\prime}\left(r,-\delta_{1 \rho}, z\right) d r, \quad \Re \mu<1+1 / \rho .
\end{aligned}
$$

Thus, we have obtained the statement of the theorem for the third case.

Case 4. Consider now the case $1 / 2<\rho \leqslant 1, \delta_{1 \rho}=\delta_{2 \rho}=\pi$. In this case the segments $\Gamma_{1}=\{\zeta$ : $\arg \zeta=-2 \pi, \varepsilon \leqslant|\zeta| \leqslant 1+\epsilon\}$ and $\Gamma_{2}=\{\zeta: \arg \zeta=0, \varepsilon \leqslant|\zeta| \leqslant 1+\epsilon\}$ of the auxiliary contour $\Gamma$ (see Figure 2) run along the positive part of a real axis. However, the argument of these segments differs by the angle $2 \pi$. Thus, the segment $\Gamma_{1}$ passes through a singular point $\zeta^{\prime}=1\left(\left|\zeta^{\prime}\right|=1, \arg \zeta^{\prime}=-2 \pi\right)$, and the segment $\Gamma_{2}$ passes through a singular point $\zeta^{\prime \prime}=1\left(\left|\zeta^{\prime \prime}\right|=1\right.$, $\left.\arg \zeta^{\prime \prime}=0\right)$. Consequently, the contour $\Gamma$ must be changed so as to bypass these two points leaving them outside the contour.

We consider the auxiliary contour $\Gamma^{\prime \prime \prime}$ (Figure 5) consisting of the arc of the circle $C_{\epsilon}$ with the center at the origin of coordinates and radius $1+\epsilon, \epsilon>0$, the segment $A B$, the arc of the circle $C_{1 \varepsilon_{1}}^{\prime \prime}$ with the center in the point $\zeta^{\prime \prime}=1\left(\left|\zeta^{\prime \prime}\right|=1, \arg \zeta^{\prime \prime}=0\right)$ and radius $0<\varepsilon_{1}<\min (1, \epsilon)$ defined by the expression (69), the segment $C D$, the arc of the circle $C_{\varepsilon}$ with the center at the origin of coordinates and radius $0<\varepsilon<1-\varepsilon_{1}$, the segment $E F$, the arc of the circle $C_{1 \varepsilon_{1}}^{\prime}$ with the center in the point $\zeta^{\prime}=1\left(\left|\zeta^{\prime}\right|=1, \arg \zeta^{\prime}=-2 \pi\right)$ and radius $0<\varepsilon_{1}<\min (1, \epsilon)$ having the form (61) and the segment $G H$. The contour is traversed in a positive direction. As a result, the contour $\Gamma^{\prime \prime \prime}$ is written in the form 


$$
\Gamma^{\prime \prime \prime}=\left\{\begin{array}{l}
C_{\epsilon}=\{\zeta:-2 \pi \leqslant \arg \zeta \leqslant 0,|\zeta|=1+\epsilon\}, \\
A B=\left\{\zeta: \arg \zeta=0,1+\epsilon \geqslant|\zeta| \geqslant 1+\varepsilon_{1}\right\}, \\
C_{1 \varepsilon_{1}}^{\prime \prime}=\left\{\begin{array}{c}
\arg \zeta=\arctan \left(\frac{\tau \sin \psi}{\tau \cos \psi+1}\right)+k \pi, \\
\zeta: \quad|\zeta|=\sqrt{\tau^{2}+2 \tau \cos \psi+1}, \\
0 \geqslant \psi \geqslant-\pi, \tau=\varepsilon_{1}, k=0
\end{array}\right\}, \\
C D=\left\{\zeta: \arg \zeta=0,1-\varepsilon_{1} \geqslant|\zeta| \geqslant \varepsilon\right\}, \\
C_{\varepsilon}=\{\zeta: 0 \geqslant \arg \zeta \geqslant-2 \pi,|\zeta|=\varepsilon\}, \\
E F=\left\{\zeta: \arg \zeta=-2 \pi, \varepsilon \leqslant|\zeta| \leqslant 1-\varepsilon_{1}\right\}, \\
C_{1 \varepsilon_{1}}^{\prime}=\left\{\begin{array}{c}
\arg \zeta=\arctan \left(\frac{\tau \sin \psi}{\tau \cos \psi+1}\right)+k \pi, \\
\zeta: \quad|\zeta|=\sqrt{\tau^{2}+2 \tau \cos \psi+1}, \\
-\pi \geqslant \psi \geqslant-2 \pi, \tau=\varepsilon_{1}, k=-2,
\end{array}\right\}, \\
G H=\left\{\zeta: \arg \zeta=-2 \pi, 1+\varepsilon_{1} \leqslant|\zeta| \leqslant 1+\epsilon\right\} .
\end{array}\right.
$$

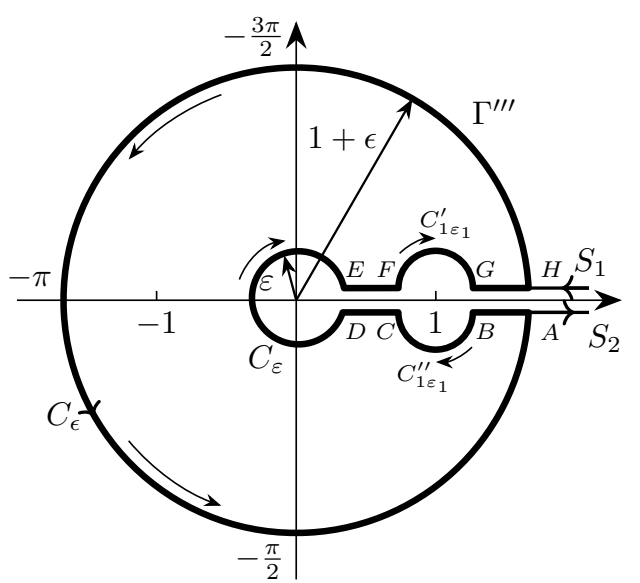

Figure 5. The auxiliary contour of integration $\Gamma^{\prime \prime \prime}$.

Next, we consider an auxiliary integral

$$
I^{\prime \prime \prime}=\int_{\Gamma^{\prime \prime \prime}} \frac{\phi_{\rho, \mu}(\zeta, z)}{\zeta-1} d \zeta,
$$

where $\phi_{\rho, \mu}(\zeta, z)$ is defined by (13). By calculating this integral directly we obtain

$$
\begin{aligned}
I^{\prime \prime \prime}=\int_{\Gamma^{\prime \prime \prime}} \frac{\phi_{\rho, \mu}(\zeta, z)}{\zeta-1} d \zeta=\int_{C_{\varepsilon}} \frac{\phi_{\rho, \mu}(\zeta, z)}{\zeta-1} d \zeta+\int_{A B} \frac{\phi_{\rho, \mu}(\zeta, z)}{\zeta-1} d \zeta+\int_{C_{1 \varepsilon_{1}}^{\prime \prime}} \frac{\phi_{\rho, \mu}(\zeta, z)}{\zeta-1} d \zeta \\
\quad+\int_{C D} \frac{\phi_{\rho, \mu}(\zeta, z)}{\zeta-1} d \zeta+\int_{C_{\varepsilon}} \frac{\phi_{\rho, \mu}(\zeta, z)}{\zeta-1} d \zeta+\int_{E F} \frac{\phi_{\rho, \mu}(\zeta, z)}{\zeta-1} d \zeta+\int_{C_{1 \varepsilon_{1}}^{\prime}} \frac{\phi_{\rho, \mu}(\zeta, z)}{\zeta-1} d \zeta \\
\quad+\int_{G H} \frac{\phi_{\rho, \mu}(\zeta, z)}{\zeta-1} d \zeta=I_{C_{\varepsilon}}+I_{A B}^{\prime \prime \prime}+I_{C_{1 \varepsilon_{1}}^{\prime \prime \prime}}^{\prime \prime \prime}+I_{C D}^{\prime \prime \prime}+I_{C_{\varepsilon}}+I_{E F}^{\prime \prime \prime}+I_{C_{1 \varepsilon_{1}}^{\prime \prime \prime}}^{\prime \prime \prime}+I_{G H}^{\prime \prime \prime} \cdot
\end{aligned}
$$


We immediately note that inside the region bounded by the contour $\Gamma^{\prime \prime \prime}$, the function $\phi_{\rho, \mu}(\zeta, z) /(\zeta-1)$ is an analytical function of the complex variable $\zeta$. Consequently, according to the Cauchy integral theorem,

$$
I_{C_{\epsilon}}+I_{A B}^{\prime \prime \prime}+I_{C_{1 \varepsilon_{1}}^{\prime \prime \prime}}^{\prime \prime \prime}+I_{C D}^{\prime \prime \prime}+I_{C_{\varepsilon}}+I_{E F}^{\prime \prime \prime}+I_{C_{1 \varepsilon_{1}}^{\prime \prime \prime}}^{\prime \prime \prime}+I_{G H}^{\prime \prime \prime}=0
$$

From this it directly follows that

$$
I_{C_{\epsilon}}=-I_{A B}^{\prime \prime \prime}-I_{C_{1 \varepsilon_{1}}^{\prime \prime \prime}}^{\prime \prime \prime}-I_{C D}^{\prime \prime \prime}-I_{C_{\varepsilon}}-I_{E F}^{\prime \prime \prime}-I_{C_{1 \varepsilon_{1}}^{\prime \prime \prime}}^{\prime \prime \prime}-I_{G H}^{\prime \prime \prime}
$$

Next, we calculate each integral on the right-hand side of this expression. However, these integrals were already calculated by us earlier. The integral $I_{A B}^{\prime \prime \prime}$ corresponds to the integral $I_{A B}^{\prime \prime}$. Therefore, using (73) we obtain

$$
I_{A B}^{\prime \prime \prime}=\int_{1+\epsilon}^{1+\varepsilon_{1}} K_{\rho, \mu}^{\prime}(r, \pi, z) d r .
$$

The integral $I_{C D}^{\prime \prime \prime}$ corresponds to the integral $I_{C D}^{\prime \prime}$. Using (74), we obtain

$$
I_{C D}^{\prime \prime \prime}=\int_{1-\varepsilon_{1}}^{\varepsilon} K_{\rho, \mu}^{\prime}(r, \pi, z) d r
$$

The integral $I_{C_{1 \varepsilon_{1}}^{\prime \prime \prime}}^{\prime \prime \prime}$ corresponds to the integral $I_{C_{1 \varepsilon_{1}}^{\prime \prime}}^{\prime \prime}$. Using (76)

$$
I_{C_{1 \varepsilon_{1}}^{\prime \prime}}^{\prime \prime \prime}=-\int_{-\pi}^{0} P_{\rho, \mu}^{\prime}\left(\varepsilon_{1}, \psi, 0, z\right) d \psi
$$

The integral $I_{C_{\varepsilon}}$ was considered by us when dealing with case 1 . Using (42) for the case considered, we obtain

$$
\lim _{\varepsilon \rightarrow 0} I_{C_{\varepsilon}}=0, \quad \Re \mu<1+1 / \rho .
$$

The integral $I_{E F}^{\prime \prime \prime}$ corresponds to the integral $I_{C D}^{\prime}$. Using (59), we obtain

$$
I_{E F}^{\prime \prime \prime}=\int_{\varepsilon}^{1-\varepsilon_{1}} K_{\rho, \mu}^{\prime}(r,-\pi, z) d r .
$$

The integral $I_{G H}^{\prime \prime \prime}$ corresponds to the integral $I_{E F}^{\prime}$. Therefore, using (60) we obtain

$$
I_{G H}^{\prime \prime \prime}=\int_{1+\varepsilon_{1}}^{1+\epsilon} K_{\rho, \mu}^{\prime}(r,-\pi, z) d r .
$$

The integral $I_{C_{1 \varepsilon_{1}}^{\prime \prime \prime}}^{\prime \prime \prime}$ corresponds to the integral $I_{C_{1 \varepsilon_{1}}^{\prime}}^{\prime}$. Using (67), we obtain

$$
I_{C_{1 \varepsilon_{1}}^{\prime \prime \prime}}^{\prime \prime \prime}=-\int_{-2 \pi}^{-\pi} P_{\rho, \mu}^{\prime}\left(\varepsilon_{1}, \psi,-2, z\right) d \psi
$$

We get back to the expression (78) and let $\varepsilon \rightarrow 0$ in it. Note that the integrals $I_{A B^{\prime}}^{\prime \prime \prime} I_{C_{1 \varepsilon_{1}}^{\prime \prime \prime}}^{\prime \prime \prime} I_{C_{1 \varepsilon_{1}}^{\prime \prime \prime}}^{\prime \prime}, I_{G H}^{\prime \prime \prime}$ do not depend on $\varepsilon$. Therefore, with such a passage to the limit, they do not change. Taking into account the above expressions for the integrals, the sum (78) takes the form 


$$
\begin{aligned}
I_{C_{\varepsilon}} & =-\int_{1+\epsilon}^{1+\varepsilon_{1}} K_{\rho, \mu}^{\prime}(r, \pi, z) d r+\int_{-\pi}^{0} P_{\rho, \mu}^{\prime}\left(\varepsilon_{1}, \psi, 0, z\right) d \psi-\lim _{\varepsilon \rightarrow 0} \int_{1-\varepsilon_{1}}^{\varepsilon} K_{\rho, \mu}^{\prime}(r, \pi, z) d r \\
& -\lim _{\varepsilon \rightarrow 0} \int_{\varepsilon}^{1-\varepsilon_{1}} K_{\rho, \mu}^{\prime}(r,-\pi, z) d r+\int_{-2 \pi}^{-\pi} P_{\rho, \mu}^{\prime}\left(\varepsilon_{1}, \psi,-2, z\right) d \psi-\int_{1+\varepsilon_{1}}^{1+\epsilon} K_{\rho, \mu}^{\prime}(r,-\pi, z) d r, \quad \Re \mu<1+1 / \rho .
\end{aligned}
$$

Performing the passage to the limit in this expression $\varepsilon \rightarrow 0$ we obtain

$$
\begin{aligned}
I_{C_{\varepsilon}}=-\int_{1+\epsilon}^{1+\varepsilon_{1}} K_{\rho, \mu}^{\prime}(r, \pi, z) d r & +\int_{-\pi}^{0} P_{\rho, \mu}^{\prime}\left(\varepsilon_{1}, \psi, 0, z\right) d \psi-\int_{1-\varepsilon_{1}}^{0} K_{\rho, \mu}^{\prime}(r, \pi, z) d r-\int_{0}^{1-\varepsilon_{1}} K_{\rho, \mu}^{\prime}(r,-\pi, z) d r \\
& +\int_{-2 \pi}^{-\pi} P_{\rho, \mu}^{\prime}\left(\varepsilon_{1}, \psi,-2, z\right) d \psi-\int_{1+\varepsilon_{1}}^{1+\epsilon} K_{\rho, \mu}^{\prime}(r,-\pi, z) d r, \quad \Re \mu<1+1 / \rho . \quad
\end{aligned}
$$

Now we consider the Mittag-Leffler (37). Taking into account that in the studied case $\delta_{1 \rho}=\delta_{2 \rho}=\pi$ we get

$$
E_{\rho, \mu}(z)=\int_{\gamma_{\zeta}} \frac{\phi_{\rho, \mu}(\zeta, z)}{\zeta-1} d \zeta
$$

where the contour $\gamma_{\zeta}$ takes the form

$$
\gamma_{\zeta}=\left\{\begin{array}{l}
S_{1}=\{\zeta: \arg \zeta=-2 \pi,|\zeta| \geqslant 1+\epsilon\} \\
C_{\varepsilon}=\{\zeta:-2 \pi \leqslant \arg \zeta \leqslant 0,|\zeta|=1+\epsilon \\
S_{2}=\{\zeta: \arg \zeta=0,|\zeta| \geqslant 1+\epsilon\}
\end{array}\right.
$$

and the condition (2) is written in the form $\frac{\pi}{2 \rho}<\arg z<-\frac{\pi}{2 \rho}+2 \pi$.

Directly calculating this integral and representing the complex number $\zeta$ in the form $\zeta=r e^{i \varphi}$ we have

$$
\begin{aligned}
E_{\rho, \mu}(z)=\int_{S_{1}} \frac{\phi_{\rho, \mu}(\zeta, z)}{\zeta-1} d \zeta+\int_{C_{\epsilon}} \frac{\phi_{\rho, \mu}(\zeta, z)}{\zeta-1} d \zeta+\int_{S_{2}} \frac{\phi_{\rho, \mu}(\zeta, z)}{\zeta-1} d \zeta= \\
\quad=\left.\int_{\infty}^{1+\epsilon} \frac{\phi_{\rho, \mu}\left(r e^{i \varphi}, z\right)}{r e^{i \varphi}-1} e^{i \varphi} d r\right|_{\varphi=-\pi-\pi}+I_{C_{\epsilon}}+\left.\int_{1+\epsilon}^{\infty} \frac{\phi_{\rho, \mu}\left(r e^{i \varphi}, z\right)}{r e^{i \varphi}-1} e^{i \varphi} d r\right|_{\varphi=\pi-\pi} \\
\quad=\int_{\infty}^{1+\epsilon} \frac{\phi_{\rho, \mu}\left(r e^{i(-\pi-\pi)}, z\right)}{r e^{i(-\pi-\pi)}-1} e^{i(-\pi-\pi)} d r+I_{C_{\epsilon}}+\int_{1+\epsilon}^{\infty} \frac{\phi_{\rho, \mu}\left(r e^{i(\pi-\pi)}, z\right)}{r e^{i(\pi-\pi)}-1} e^{i(\pi-\pi)} d r .
\end{aligned}
$$

Now using here (56) we obtain that the expression may be represented in the form

$$
E_{\rho, \mu}(z)=\int_{\infty}^{1+\epsilon} K_{\rho, \mu}^{\prime}(r,-\pi, z) d r+I_{C_{\epsilon}}+\int_{1+\epsilon}^{\infty} K_{\rho, \mu}^{\prime}(r, \pi, z) d r
$$

Now here we make use of the representation (79) for the integral $I_{C_{\epsilon}}$. As a result, we obtain 


$$
\begin{aligned}
& E_{\rho, \mu}(z)=\int_{\infty}^{1+\epsilon} K_{\rho, \mu}^{\prime}(r,-\pi, z) d r-\int_{1+\epsilon}^{1+\varepsilon_{1}} K_{\rho, \mu}^{\prime}(r, \pi, z) d r+\int_{-\pi}^{0} P_{\rho, \mu}^{\prime}\left(\varepsilon_{1}, \psi, 0, z\right) d \psi-\int_{1-\varepsilon_{1}}^{0} K_{\rho, \mu}^{\prime}(r, \pi, z) d r \\
&-\int_{0}^{1-\varepsilon_{1}} K_{\rho, \mu}^{\prime}(r,-\pi, z) d r+\int_{-2 \pi}^{-\pi} P_{\rho, \mu}^{\prime}\left(\varepsilon_{1}, \psi,-2, z\right) d \psi-\int_{1+\varepsilon_{1}}^{1+\epsilon} K_{\rho, \mu}^{\prime}(r,-\pi, z) d r+\int_{1+\epsilon}^{\infty} K_{\rho, \mu}^{\prime}(r, \pi, z) d r \\
&=\int_{0}^{1-\varepsilon_{1}}\left(K_{\rho, \mu}^{\prime}(r, \pi, z)-K_{\rho, \mu}^{\prime}(r,-\pi, z)\right) d r+\int_{1+\varepsilon_{1}}^{\infty}\left(K_{\rho, \mu}^{\prime}(r, \pi, z)-K_{\rho, \mu}^{\prime}(r,-\pi, z)\right) d r \\
&+\int_{-\pi}^{0} P_{\rho, \mu}^{\prime}\left(\varepsilon_{1}, \psi, 0, z\right) d \psi+\int_{-2 \pi}^{-\pi} P_{\rho, \mu}^{\prime}\left(\varepsilon_{1}, \psi,-2, z\right) d \psi
\end{aligned}
$$

In the expression derived there is the difference of $K_{\rho, \mu}^{\prime}(r, \pi, z)-K_{\rho, \mu}^{\prime}(r,-\pi, z)$. We transform this difference. For this we use the representation (57) for the function $K_{\rho, \mu}^{\prime}(r, \delta, z)$. As a result, we obtain

$$
\begin{aligned}
K_{\rho, \mu}^{\prime}(r, \delta, z) & -K_{\rho, \mu}^{\prime}(r,-\delta, z) \\
& =\frac{\rho}{2 \pi i} \frac{\exp \left\{(z r)^{\rho} e^{-i \pi \rho} \cos (\rho \delta)\right\}\left(z r e^{-i \pi}\right)^{\rho(1-\mu)}}{r^{2}+2 r \cos \delta+1}\left(e^{i \eta(r, \delta, z)}\left(r+e^{i \delta}\right)-e^{i \eta(r,-\delta, z)}\left(r+e^{-i \delta}\right)\right) .
\end{aligned}
$$

From the definition for the function $\eta(r, \delta, z)(10)$ it follows that it is an odd function relative to the parameter $\delta$

$$
\eta(r,-\delta, z)=-\eta(r, \delta, z)
$$

In the total, we have

$$
\begin{aligned}
& K_{\rho, \mu}^{\prime}(r, \delta, z)-K_{\rho, \mu}^{\prime}(r,-\delta, z) \\
& \quad=\frac{\rho}{2 \pi i} \frac{\exp \left\{(z r)^{\rho} e^{-i \pi \rho} \cos (\rho \delta)\right\}\left(z r e^{-i \pi}\right)^{\rho(1-\mu)}}{r^{2}+2 r \cos \delta+1}\left(e^{i \eta(r, \delta, z)}\left(r+e^{i \delta}\right)-e^{-i \eta(r, \delta, z)}\left(r+e^{-i \delta}\right)\right) \\
& \frac{\rho}{2 \pi i} \frac{\exp \left\{(z r)^{\rho} e^{-i \pi \rho} \cos (\rho \delta)\right\}\left(z r e^{-i \pi}\right)^{\rho(1-\mu)}}{r^{2}+2 r \cos \delta+1}\left(r\left(e^{i \eta(r, \delta, z)}-e^{-i \eta(r, \delta, z)}\right)+\left(e^{i(\eta(r, \delta, z)+\delta)}-e^{-i(\eta(r, \delta, z)+\delta)}\right)\right) \\
& =\frac{\rho}{2 \pi i} \frac{\exp \left\{(z r)^{\rho} e^{-i \pi \rho} \cos (\rho \delta)\right\}\left(z r e^{-i \pi}\right)^{\rho(1-\mu)}}{r^{2}+2 r \cos \delta+1}(r \sin (\eta(r, \delta, z))+\sin (\eta(r, \delta, z)+\delta))=K_{\rho, \mu}(r, \delta, z) .
\end{aligned}
$$

Here the function $K_{\rho, \mu}(r, \delta, z)$ was obtained by us earlier and has the form (19). As a result, the expression (81) takes the form

$$
E_{\rho, \mu}(z)=\int_{0}^{1-\varepsilon_{1}} K_{\rho, \mu}(r, \pi, z) d r+\int_{1+\varepsilon_{1}}^{\infty} K_{\rho, \mu}(r, \pi, z) d r+\int_{-\pi}^{0} P_{\rho, \mu}^{\prime}\left(\varepsilon_{1}, \psi, 0, z\right) d \psi+\int_{-2 \pi}^{-\pi} P_{\rho, \mu}^{\prime}\left(\varepsilon_{1}, \psi,-2, z\right) d \psi .
$$

The derived expression proves the theorem completely.

The proved theorem shows that in the representation (3) at the values of parameters $\rho, \delta_{1 \rho}, \delta_{2 \rho}$ satisfying the conditions (32) the integral over the arc of the circle $C_{\epsilon}$ of the contour $\gamma_{\zeta}$ can be replaced with integration with respect to the segments $\Gamma_{1}$ and $\Gamma_{2}$ of the contour (39). Such a transition makes it possible to replace the contour integral with one improper integral over the real variable of the complex-valued function, which simplifies the use and study of the Mittag-Leffler function. The integral representation of the Mittag-Leffler function obtained in this theorem will be called the integral representation " $\mathrm{B}$ ".

Consider in detail the differences between two derived representations. The integral representation " $\mathrm{A}$ " (see (7)) of the Mittag-Leffler function consists of two summands. The first summand corresponds to the sum of integrals along the half-lines $S_{1}$ and $S_{2}$ and the second summand is the integral along the arc 
of a circle $C_{\epsilon}$. Such a representation is not convenient in analytical studies of the Mittag-Leffler function since one has to deal with two integrals. The representation " $\mathrm{B}$ " turns out to be more convenient and it consists of one improper integral (see (33)). However, this convenience is fraught with the appearance of constraints imposed on values of the parameters of the function $E_{\rho, \mu}(z)$. As it was shown in Theorem 3 , the representation "B" is true only at values $\Re \mu<1+1 / \rho$. If $\Re \mu \geqslant 1+1 / \rho$, then it is necessary to use the representation " $\mathrm{A}$ " which is the main integral representation for the Mittag-Leffler function. It should also be noted that in the representation " $\mathrm{B}$ " with the parameter values $1 / 2<\rho \leqslant 1$ and values $\delta_{1 \rho}=\pi$ or $\delta_{2 \rho}=\pi$ the segments $\Gamma_{1}$ or $\Gamma_{2}$ of the auxiliary contour $\Gamma$ (see Figure 2) pass through the pole $\zeta=1$. This leads to the need to deform the contour of integration so as to bypass the pole. As a result, in integral representations (34), (35), (36) there are summands describing the pole bypass along the arcs of the circle $C_{1 \varepsilon_{1}}^{\prime}$ and $C_{1 \varepsilon_{1}}^{\prime \prime}$. In addition, one has to split the integrals along the half-lines $\Gamma_{1}+S_{1}$ and $\Gamma_{2}+S_{2}$ into parts to exclude the sections corresponding to the integrals along the arcs of the circle $C_{1 \varepsilon_{1}}^{\prime}$ and $C_{1 \varepsilon_{1}}^{\prime \prime}$. All this leads to the appearance of additional terms in the corresponding integral representations. It should also be noted that at parameter values $1 / 2<\rho \leqslant 1, \delta_{1 \rho}=\delta_{2 \rho}=\pi$ in the representation (36) the integral $\int_{0}^{1-\varepsilon_{1}} K_{\rho, \mu}(r, \pi, z) d r$ corresponds to the sum of integrals along the segments $C D$ and $E F$ the contour of integration $\Gamma^{\prime \prime \prime}$ (see Figure 5) the integral $\int_{1+\varepsilon_{1}}^{\infty} K_{\rho, \mu}(r, \pi, z) d r$ corresponds to the sum of integrals along $A B+S_{2}$ and $G H+S_{1}$. As one can see, the specified sections of the integration contour go along the positive part of a real axis. As it was shown in the Lemma 1 at values of parameters $1 / 2<\rho<1$ or $\rho=1$ and $\Im \mu \neq 0$ or $\rho=1, \Im \mu=0$ and $\Re \mu$ is not an integer, the point $\zeta=0$ is a branch point of the integrand (37). Consequently, the indicated sections of the integration contour will go along different sides of the cut of the complex plane $\zeta$. As a result, $\int_{0}^{1-\varepsilon_{1}} K_{\rho, \mu}(r, \pi, z) d r \neq 0$ and $\int_{1+\varepsilon_{1}}^{\infty} K_{\rho, \mu}(r, \pi, z) d r \neq 0$. In case, if $\rho=1, \Im \mu=0$ and $\Re \mu$ is an integer, Lemma 1 shows that in this case the point $\zeta=0$ is a regular point. Consequently, the segments $C D$ and $E F$, as well as the half-lines $A B+S_{2}$ and $G H+S_{1}$ will go along one straight line of the complex plane, but in different directions, and arcs of the circles $C_{1 \varepsilon_{1}}^{\prime}$ and $C_{1 \varepsilon_{1}}^{\prime \prime}$ will close. As a result, we obtain $\int_{0}^{1-\varepsilon_{1}} K_{1, \mu}(r, \pi, z) d r=0$ and $\int_{1+\varepsilon_{1}}^{\infty} K_{1, \mu}(r, \pi, z) d r=0$, and the sum of integrals $\int_{-\pi}^{0} P_{1, \mu}^{\prime}\left(\varepsilon_{1}, \psi, 0, z\right) d \psi+\int_{-2 \pi}^{-\pi} P_{1, \mu}^{\prime}\left(\varepsilon_{1}, \psi,-2, z\right) d \psi$ corresponds to the integral over the closed contour and will be equal to the residue in the point $\zeta=1$. Thus, we come to the condition of Corollary 2.

The formulas of the integral representation obtained in Theorem 3 are rather lengthy. They can be simplified and reduced to a simpler form. The representation " $\mathrm{B}$ " takes the simplest form in the case when $\delta_{1 \rho}$ and $\delta_{2 \rho}$ coincide, i.e., $\delta_{1 \rho}=\delta_{2 \rho}=\delta_{\rho}$. We will formulate the result obtained in the form of a corollary.

Corollary 3. For any real $\rho>1 / 2$, any complex $\mu$ and $z$ satisfying the conditions $\Re \mu<1+\frac{1}{\rho}$ and

$$
\frac{\pi}{2 \rho}-\delta_{\rho}+\pi<\arg z<-\frac{\pi}{2 \rho}+\delta_{\rho}+\pi,
$$

the Mittag-Leffler function can be represented in the form:

1. at any real $\delta_{\rho}$ satisfying the conditions $\frac{\pi}{2 \rho}<\delta_{\rho} \leqslant \frac{\pi}{\rho}$ if $\rho>1$ and $\frac{\pi}{2 \rho}<\delta_{\rho}<\pi$ if $1 / 2<\rho \leqslant 1$

$$
E_{\rho, \mu}(z)=\int_{0}^{\infty} K_{\rho, \mu}\left(r, \delta_{\rho}, z\right) d r,
$$

where $K_{\rho, \mu}\left(r, \delta_{\rho}, z\right)$ has the form (19).

2. at $\delta_{\rho}=\frac{\pi}{\rho}$ and $\rho>1$

$$
E_{\rho, \mu}(z)=\int_{0}^{\infty} K_{\rho, \mu}(r, z) d r
$$

where $K_{\rho, \mu}(r, z)$ has the form (21). 
Proof. (1) According to Theorem 3 the representation (33) is true at values $\delta_{1 \rho}$ and $\delta_{2 \rho}$ satisfying the conditions (32). In this corollary it is assumed that $\delta_{1 \rho}=\delta_{2 \rho}=\delta_{\rho}$. In view of this assumption, the conditions (32) take the form $\frac{\pi}{2 \rho}<\delta_{\rho} \leqslant \frac{\pi}{\rho}$ if $\rho>1$ and $\frac{\pi}{2 \rho}<\delta_{\rho}<\pi$ if $1 / 2<\rho \leqslant 1$. The representation (33) will be written in the form

$$
E_{\rho, \mu}(z)=\int_{0}^{\infty} K_{\rho, \mu}\left(r,-\delta_{\rho}, \delta_{\rho}, z\right) d r .
$$

From this it is clear that it is necessary to consider how the kernel function $K_{\rho, \mu}\left(r,-\delta_{\rho}, \delta_{\rho}, z\right)$ is transformed at the parameter values specified. This issue was already considered when proving item 1 of Corollary 1 (see (25)). It was shown there that

$$
\begin{aligned}
K_{\rho, \mu}\left(r,-\delta_{\rho}, \delta_{\rho}, z\right) & =K_{\rho, \mu}\left(r, \delta_{\rho}, z\right)= \\
& =\frac{\rho}{\pi}\left(z r e^{-i \pi}\right)^{\rho(1-\mu)} \exp \left\{\left(z r e^{-i \pi}\right)^{\rho} \cos \left(\rho \delta_{\rho}\right)\right\} \frac{r \sin \left(\eta\left(r, \delta_{\rho}, z\right)\right)+\sin \left(\eta\left(r, \delta_{\rho}, z\right)+\delta_{\rho}\right)}{r^{2}+2 r \cos \delta_{\rho}+1} .
\end{aligned}
$$

The first part of the corollary is proved.

(2) Consider the case $\delta_{\rho}=\pi / \rho$. Note immediately that the value of an angle $\delta_{\rho}$ cannot exceed $\pi$. Consequently, this case is implemented only at values $\rho \geqslant 1$. However, the value $\rho=1$ should be excluded from consideration. In fact, at $\rho=1$ the segments $\Gamma_{1}$ and $\Gamma_{2}$ of the auxiliary contour $\Gamma$ (see Figure 2) pass through the pole $\zeta=1$. Consequently, it is necessary to deform the contour $\Gamma$ so as to bypass this pole. However, this has already been done by us in item 4 of Theorem 3. By putting the value $\delta_{\rho}=\pi / \rho$ in the representation (83) we obtain

$$
E_{\rho, \mu}(z)=\int_{0}^{\infty} K_{\rho, \mu}(r, \pi / \rho, z) d r .
$$

Using the definition of the function $\eta(r, \psi, z)$ (see (10)) we obtain $\eta(r, \pi / \rho, z)=(1-\mu) \pi$. Now using this result when calculating the kernel function $K_{\rho, \mu}(r, \pi / \rho, z)$ and introducing the notation $K_{\rho, \mu}(r, \pi / \rho, z) \equiv K_{\rho, \mu}(r, z)$ we obtain

$$
E_{\rho, \mu}(z)=\int_{0}^{\infty} K_{\rho, \mu}(r, z) d r
$$

where $K_{\rho, \mu}(r, z)$ has the form (21).

\section{Conclusions}

It has been shown in the paper that when passing from the integral representation formulated in Theorem 1 to integration over real variables, the integral representation of the Mittag-Leffler function can be written in two forms: the representation " $A$ " and " $B$ ". The integral representation " $A$ " was given in Theorem 2 and the representation " $B$ " in Theorem 3. Each of these representations has its own advantages and drawbacks. The representation " $\mathrm{A}$ " is true for any complex $\mu$ and at values $1 / 2<\rho \leqslant 1$ there is no need to bypass a pole in the point $\zeta=1$. This excludes the necessity to consider particular cases and greatly simplifies the presentation itself. These facts are the advantages of the representation " $\mathrm{A}$ ". The disadvantages of this representation include the fact that it consists of the sum of two integrals: improper and definite. This leads to certain difficulties when working with this representation, since one has to study the behavior of these two integrals. The representation " $\mathrm{B}$ " is valid only for the parameter values $\mu$ satisfying the condition $\Re \mu<1+1 / \rho$. Particular cases also arise at values $1 / 2<\rho \leqslant 1$ in which one has to bypass a singular point $\zeta=1$. That is why, in the problems in which there is a necessity to investigate these particular cases this form of representation is not very convenient. These facts are the 
disadvantage of the representation " $\mathrm{B}$ ". In all other cases the representation " $\mathrm{B}$ " consists of one improper integral and turns out to be more convenient in use than the representation " $\mathrm{A}$ ". This is the advantage of the representation " $\mathrm{B}$ ".

The forms of the representations " $\mathrm{A}$ " and " $\mathrm{B}$ " obtained in Theorems 2 and 3 are given for the case of four parameters $\rho, \mu, \delta_{1 \rho}, \delta_{2 \rho}$. The parameters $\rho$ and $\mu$ are referred to the Mittag-Leffler function directly, and parameters $\delta_{1 \rho}$ and $\delta_{2 \rho}$ describe the integration contour. As a result, in the general case, the integral representations " $\mathrm{A}$ " and " $\mathrm{B}$ " of the Mittag-Leffler function turn out to be four-parameter. For definiteness, we call this four-parameter description parametrization 1 . However, in parametrization 1 though the representations " $\mathrm{A}$ " and " $\mathrm{B}$ " of the function $E_{\rho, \mu}(z)$ have a more general form but they are awkward enough. The representations " $\mathrm{A}$ " and " $\mathrm{B}$ " take a simpler form if $\delta_{1 \rho}=\delta_{2 \rho}=\delta_{\rho}$. In this case, the integral representations " $\mathrm{A}$ " and " $\mathrm{B}$ " can be described in three parameters: $\rho, \mu, \delta_{\rho}$. We call this parametrization 2. An even simpler view of the form " $\mathrm{A}$ " and " $\mathrm{B}$ " is adopted if $\delta_{1 \rho}=\delta_{2 \rho}=\pi / \rho$. In this case integral representations of the Mittag-Leffler function can be described in two parameters $\rho$ and $\mu$. We call this case of parametrization-parametrization 3. Thus, the integral representations of the Mittag-Leffler function in the form " $\mathrm{A}$ " and parameterizations 2 and 3 are given in Corollary 1, and the representation " $\mathrm{B}$ " in parametrizations 2 and 3 in Corollary 3.

Taking into account of the geometric meaning of the parameters $\delta_{1 \rho}$ and $\delta_{2 \rho}$ one can give the geometric interpretation of three introduced parametrizations of the representations " $\mathrm{A}$ " and " $\mathrm{B}$ ". In fact, the parameters $\delta_{1 \rho}$ and $\delta_{2 \rho}$ describe an inclination angle of half-lines $S_{1}$ and $S_{2}$ in the contour $\gamma_{\zeta}$ (see Figure 1). Thus, in case if the half-lines $S_{1}$ and $S_{2}$ independently lie in the range of angles $\frac{\pi}{2 \rho}<\delta_{1 \rho} \leqslant \min \left(\pi, \frac{\pi}{\rho}\right), \quad \frac{\pi}{2 \rho}<\delta_{2 \rho} \leqslant \min \left(\pi, \frac{\pi}{\rho}\right)$, then we have parametrization 1 . If these half-lines lie symmetrically in relation to the positive part of a real axis, then we obtain parametrizations 2 and 3 . With this, parametrization 3 corresponds to the angle of inclination $\delta_{1 \rho}=\delta_{2 \rho}=\pi / \rho$.

In conclusion it should be pointed out that the representations for the function $E_{\rho, \mu}(z)$, formulated in Theorems 2 and 3 are valid for the vaues $\arg z$ satisfying the condition $\frac{\pi}{2 \rho}-\delta_{2 \rho}+\pi<\arg z<-\frac{\pi}{2 \rho}+\delta_{1 \rho}+\pi$. This constraint appears as a result of the use of the proof of Theorem 1 of the integral representation for the gamma function obtained in the work [22] (see Appendix in [22]). The presence of this constraint somewhat narrows the possibilities of using the obtained integral representations of the function $E_{\rho, \mu}(z)$. Nevertheless, it is possible to get rid of this constraint and expand the range of admissible values $\arg z$ to the entire complex plane. However, this requires additional studies that are beyond the scope of this paper.

Funding: This work was supported by the Russian Foundation for Basic Research (projects No 19-44-730005, 20-07-00655, 18-44-730004) and the Ministry of Science and Higher Education of the Russian Federation (projects No. 0830-2020-0008, RFMEFI60719X0301).

Acknowledgments: The author thanks to M. Yu. Dudikov for translation the article into English.

Conflicts of Interest: The author declares no conflict of interest.

1. Gorenflo, R.; Kilbas, A.A.; Mainardi, F.; Rogosin, S.V. Mittag-Leffler Functions, Related Topics and Applications; Springer Monographs in Mathematics; Springer: Berlin/Heidelberg, Germany, 2014; doi:10.1007/978-3-662-43930-2. [CrossRef]

2. Mittag-Leffler, M.G. Une généralization de l'intégrale de Laplace-Abel. Comp. Rend. Acad. Sci. 1903, 136, 537-539.

3. Mittag-Leffler, G. Sur la représentation analytique d'une branche uniforme d'une fonction monogène: Cinquième note. Acta Math. 1905, 29, 101-181. [CrossRef]

4. Wiman, A. Über den Fundamentalsatz in der Teorie der Funktionen Ea(x). Acta Math. 1905, $29,191-201$. [CrossRef]

5. Wiman, A. Über die Nullstellen der Funktionen Ea(x). Acta Math. 1905, 29, 217-234. [CrossRef] 
6. Humbert, P.; Agarwal, R.P. Sur la fonction de Mittag-Leffler et quelques-unes de ses généralisations. Bull. Sci. Math. II. Sér. 1953, 77, 180-185.

7. Humbert, P. Quelques résultats rélatifs à la fonction de Mittag-Leffler. C. R. Acad. Sci. 1953, 236, 1467-1468.

8. Agarwal, R.P. A propos d'une note de M. Pierre Humbert. C. R. Acad. Sci. 1953, 236, 2031-2032.

9. Djrbashian, M.M. On the integral representation of functions continuous on several rays (generalization of the Fourier integral). Izv. Akad. Nauk SSSR. Ser. Mat. 1954, 18, 427-448. (In Russian)

10. Djrbashian, M.M. On the asymptotic expansion of a function of Mittag-effler type. Akad. Nauk. Armjan. SSR Dokl. 1954, 19, 65-72. (In Russian)

11. Djrbashian, M.M. Integral Transfororms and Representation of the functions in the Complex Domain; Nauka. Glav. red. fiz.-mat. lit: Moscow, Russia, 1966; p. 672. (In Russian)

12. Popov, A.Y.; Sedletskii, A.M. Distribution of roots of Mittag-Leffler functions. J. Math. Sci. 2013, 190, $209-409$. [CrossRef]

13. Rogosin, S. The role of the Mittag-Leffler function in fractional modeling. Mathematics 2015, 3, 368-381. [CrossRef]

14. Gorenflo, R.; Mainardi, F.; Rogosin, S. Mittag-Leffler function: Properties and applications. In Handbook of Fractional Calculus with Applications. Volume 1: Basic Theory; Kochubei, A., Luchko, Y., Eds.; De Gruyter: Berlin, Germany; Boston, MA, USA, 2019; pp. 269-296. [CrossRef]

15. Bateman, H. Higher Transcendental Functions; McGraw-Hill Book Company: New York, NY, USA, 1955; Volume 3, p. 292.

16. Podlubny, I. Fractional Differential Equations; Volume 198, Mathematics in Science and Engineering; Academic Press: Amsterdam, The Netherlands, 1999; p. 340.

17. Gorenflo, R.; Loutchko, J.; Luchko, Y. Computation of the Mittag-Leffler function E $\alpha, \beta(z)$ and its derivative. Fract. Calc. Appl. Anal. 2002, 5, 491-518.

18. Seybold, H.J.; Hilfer, R. Numerical results for the generalized Mittag-Leffler function. Fract. Calc. Appl. Anal. 2005, 8, 129-139.

19. Hilfer, R.; Seybold, H.J. Computation of the generalized Mittag-Leffler function and its inverse in the complex plane. Integral Transform. Spec. Funct. 2006, 17, 637-652. [CrossRef]

20. Seybold, H.; Hilfer, R. Numerical Algorithm for Calculating the Generalized Mittag-Leffler Function. SIAM J. Numer. Anal. 2009, 47,69-88. [CrossRef]

21. Parovik, R.I. Calculation specific functions of Mittag-Leffler in the computer mathematics «Maple». Bull. KRASEC. Phys. Math. Sci. 2012, 2, 51-61. [CrossRef]

22. Saenko, V.V. An integral representation of the Mittag-Leffler function. arXiv 2020, arXiv:2001.09606.

23. Saenko, V.V. Integral representation of the Mittag-Leffler function. In Book of Abstracts 2nd International Conference on Mathematical Modeling in Appliend Sciences, BSU Belgorod-Russia (20-24 August 2019); Debbouche, A., Ed.; Belgorod State University: Belgorod, Russia, 2019; pp. 157-158.

24. Saenko, V.V. Integral Representation of the Fractional Stable Density. J. Math. Sci. 2020, 248, 51-66. [CrossRef]

25. Uchaikin, V.V.; Cahoy, D.O.; Sibatov, R.T. Fractional processes: From Poisson to branching one. Int. J. Bifurc. Chaos 2008, 18, 2717-2725. [CrossRef]

26. Uchaikin, V.; Sibatov, R.; Uchaikin, D. Memory regeneration phenomenon in dielectrics: the fractional derivative approach. Phys. Scr. 2009, T136, 014002, doi:10.1088/0031-8949/2009/T136/014002. [CrossRef]

27. Saenko, V.V. Singular points of the integral representation of the Mittag-Leffler function. arXiv 2020, 1-11, arXiv:2004.08164.

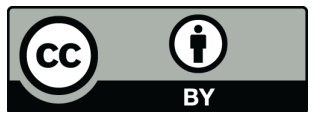

(C) 2020 by the author. Licensee MDPI, Basel, Switzerland. This article is an open access article distributed under the terms and conditions of the Creative Commons Attribution (CC BY) license (http:/ / creativecommons.org/licenses/by/4.0/). 